\title{
Agile Unternehmen
}

Zukunftsfähig in der digitalen Transformation

SAMMELBAND AUS AUSGEWÄHLTEN BLOGARTIKELN ZUM DIGITALEN WANDEL, AGILITÄT, FÜHRUNG UND ARBEIT

\section{VON DOMINIC LINDNER}

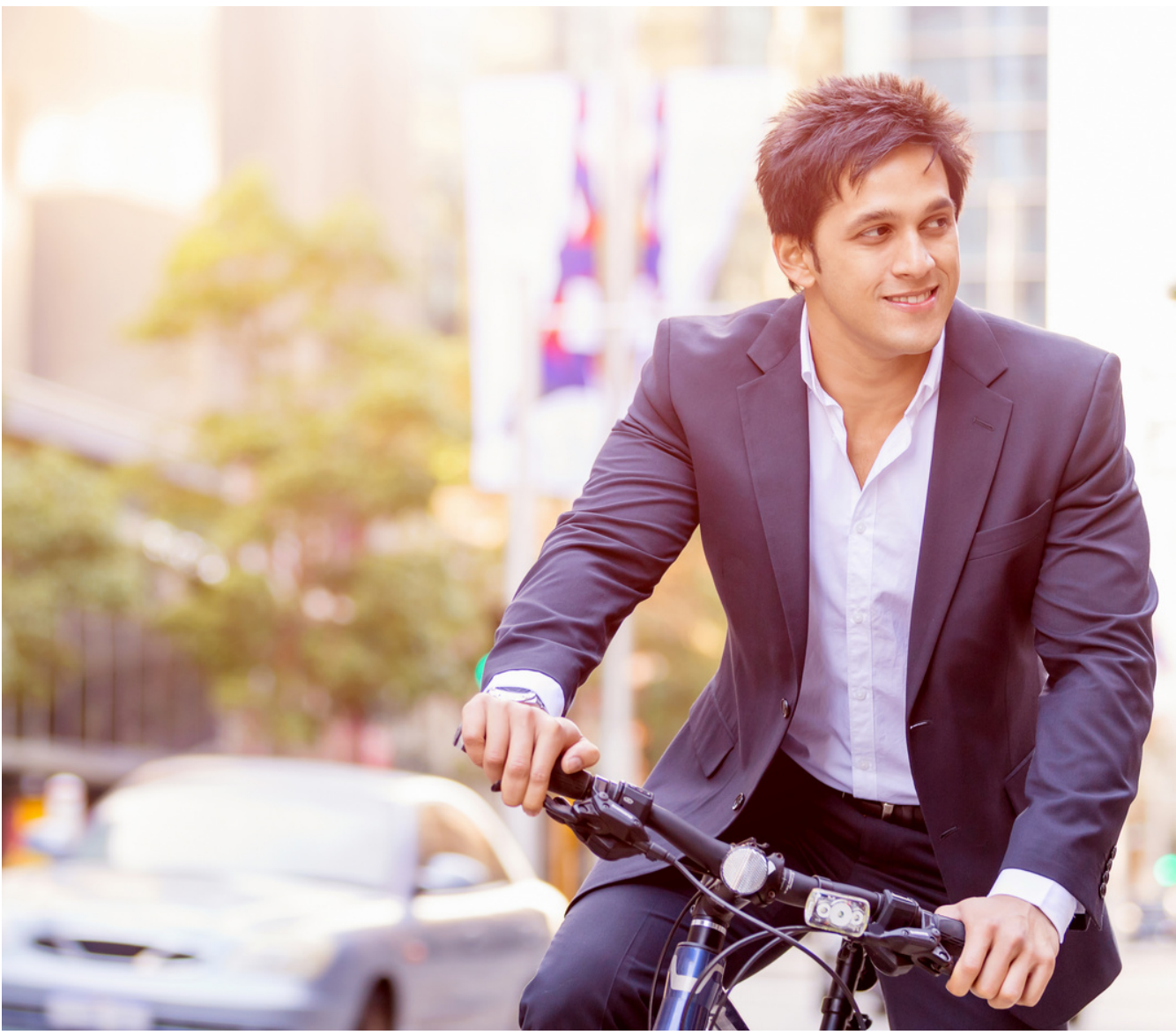




\section{Vorwort}

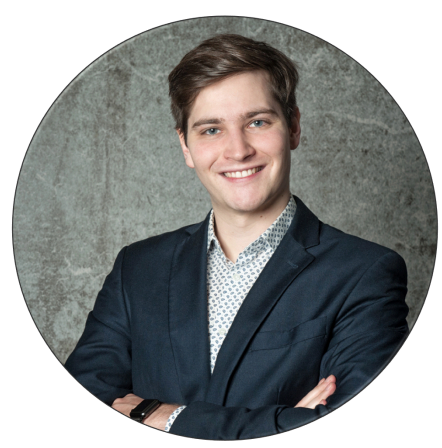

Der digitale Wandel ist im vollen Gange und wir sind gerade dabei zu begreifen, was dieser eigentlich für uns bedeutet. Oft höre ich auch meinen Vorträgen über z. B. Technostress am Arbeitsplatz Sätze wie „Wow, das war mir gar nicht so bewusst“.

Nachdem ich seit mittlerweile einem Jahr blogge, habe ich mich entschieden, ausgewählte Blogartikel zu einem Sammelband zusammenzufassen und ein Resümee zu ziehen.

Ich promoviere nebenberuflich an der FAU ErlangenNürnberg und habe in diese Blogartikel die Ergebnisse meiner Forschungsarbeit sowie die Eindrücke aus meinem Arbeitsleben einfließen lassen. Unterstützt werden diese durch Dialoge mit Experten, welche ich ebenfalls in diesem Buch eingefügt habe.

Es ist jedoch nicht immer einfach nebenberuflich zu promovieren, weswegen ich außerdem dieses Vorwort nutzen möchte, um danke zu sagen. 
In erster Linie bei allen Menschen, welche mir beruflich den Rücken freigehalten. Auch geht ein Danke an meine ehemaligen und jetzigen Führungskräfte, welche die Doktorarbeit stark unterstützen sowie meinen Professor. Weiterhin sage ich danke an die Experten um Boris Gloger, Niels Pfläging, Svenja Hofert, Valentin Nowotny und Dr. Andreas Zeuch, welche mir dabei geholfen haben, dieses Buch mit spannenden Inhalten zu füllen und mich mit ihren Werken immer wieder inspirieren. Auch möchte ich besonders die Teilnehmer meiner Roundtables hervorheben. Ich bin diesem Menschen sehr dankbar, dass mich diese seit über einem Jahr unterstützen und meine Doktorarbeit damit erst möglich machen.

Zu guter Letzt möchte ich auch meiner Freundin danken. Dafür, dass sie mir selbst nach 2 Jahren Agilität immer noch zuhört und mich auch in diesem Buch unterstützt hat und auch akzeptiert, dass ich selbst an einem sonnigen Tag wie heute gerade dieses Vorwort abtippe, anstatt mit ihr nach draußen zu gehen. Auch an meine Familie geht ein großer Dank für den emotionalen Support!

2018 werde ich das Forschungsprojekt beenden und freue mich ankündigen zu dürfen, dass es dann ein weiteres Buch geben wird, welches die Ergebnisse meiner bis dahin 3-jährigen Beobachtungen wiedergeben wird.

Ich wünsche Ihnen Freude beim Lesen und leiten Sie das Buch gern an Freunde und Bekannte weiter und zitieren Sie es gern. 


\section{Aufbau \\ AGILE UNTERNEHMEN}

Wir leben in einer komplexen Welt mit schnellen und tiefgreifenden Veränderungen. Ein wesentlicher Treiber dieser ist die exponentielle technologische Entwicklung der letzten Jahre. Diese zwingt Unternehmen sich zu verändern und mehr "Agilität" zuzulassen. In diesem EBook möchte ich die Bereiche Führung, Organisation und Arbeit im Kontext dieses Megatrends genau beleuchten.

Im ersten Kapitel zeige ich Ihnen drei Artikel zum digitalen Wandel auf. Neben Definition und einer Untersuchung, welche Herausforderungen der Wandel eigentlich an Unternehmen stellt, finden Sie auch einen Dialog mit Boris Gloger zur Skalierung von Agilität.

Im zweiten Kapitel widme ich mich dem Trend Arbeit 4.0. Neben einer Vorstellung verschiedener neuer Konzepte gibt es einen Artikel zum Einfluss der Digitalisierung auf den Arbeitsplatz sowie einen Dialog mit Dr. Andreas Zeuch.

Im dritten Kapitel dreht sich alles um die Agilität in Organisationen. Was bedeutet es eigentlich agil zu sein und warum sollten wir das tun? Neben der Beantwortung dieser Fragen rundet das Kapitel ein Dialog mit Valentin Nowotny ab.

Im letzten Kapitel geht es um die Führung und die Rolle des digitalen Leaders. Neben einer Definition von Führung gibt es einige Praxistipps für moderne Führung sowie einen spannenden Dialog mit Svenja Hofert. 


\section{SAMMELBAND \\ INHALTSVERZEICHNIS}

\section{1 | Digitaler Wandel}

2 | Was ist eigentlich der der digitale Wandel?

10 | Digitalisierung - Wandel trifft auf Tradition

17 | Dialog mit Boris Gloger

\section{4 | Arbeit 4.0}

25 | Wie definiert sich Arbeit 4.0?

33 | Die Digitalisierung am Arbeitsplatz

44 | Dialog mit Dr. Andreas Zeuch

\section{9 | Agile Organisation}

50 | Was ist eigentlich Agilität?

55 | Wie gestaltet sich agiles Management?

64 | Dialog mit Valentin Nowotny

69 | Digital Leadership

70 | Was ist Digital Leadership?

78 | Führung im technologischen Wandel

86 | Dialog mit Svenja Hofert

92 | Nachwort

93 | Bildnachweis und Diversity

94 | Impressum 


\section{Einleitung}

Wie oft schauen Sie pro Tag auf Ihr Smartphone, ohne dass es klingelt? Selbst während der Arbeitszeit ertappen wir uns dabei, unser Display regelmäßig auf neue Nachrichten zu checken, ob es nun blinkt oder nicht. Aber warum empfinden wir dieses Bedürfnis, das Telefon in die Hand zu nehmen, ohne dass wir angerufen werden oder eine Nachricht erhalten haben (Quelle: Hubspot)? Viele außerhalb der Generation Z kennen noch eine Zeit vor dem Smartphone.

Das digitale Zeitalter ist keine Modeerscheinung, sondern längst Realität. So sagt die Bitcom: Digitale Transformation ist, wenn wir Bücher nicht mehr im Laden um die Ecke kaufen, sondern online das E-Book, das sofort auf unserem Lesegerät auftaucht. Digitale Transformation ist, wenn immer mehr Menschen das Programmfernsehen links liegen lassen und lieber Filme über Streaming-Anbieter anschauen. Wenn wir kein Babyphone kaufen, sondern unser Smartphone mit einer App dafür nutzen. Digitale Transformation ist, wenn Ärzte Patienten per Video-Chat beraten oder die Röntgendiagnose von einem telemedizinischen Zentrum kommt. Und wenn uns unser Auto nach der Feier autonom nach Hause fährt und sich anschließend selbstständig einen Parkplatz sucht.

Die Umwelt von Unternehmen ist heute dynamischer und komplexer als vor 100 Jahren. Das ist eine Binsenwahrheit. Doch erst einige wenige Pionierunternehmen haben ihre Unternehmensführung den neuen Anforderungen angepasst, sodass sie der Komplexität und Dynamik deutlich erfolgreicher begegnen können als ihre Wettbewerber (Quelle: bbrt.ch). Es wird also dynamischer und komplexer. Das von Pfläging beschriebene Rote, also das Komplexe, kann nicht mehr durch alte 
Methoden gelöst werden. Dies bezeichnet er als das Blaue, was komplizierte Probleme sind.

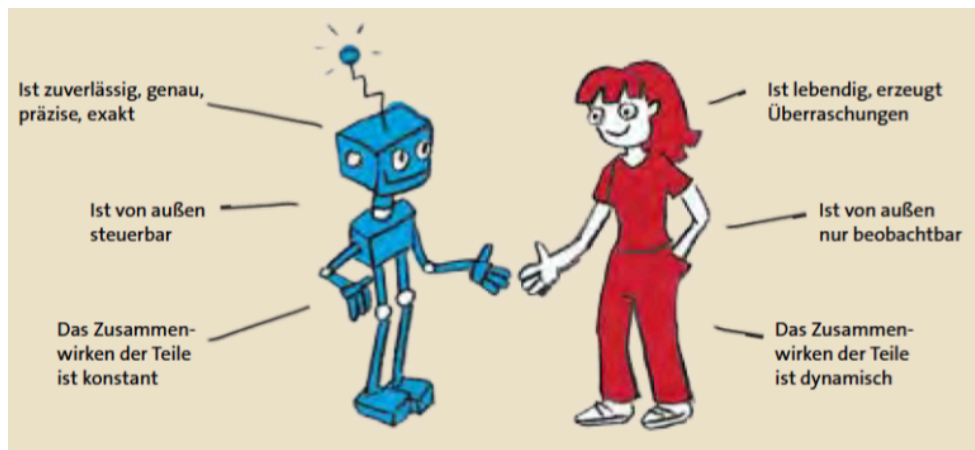

Die Domäne des Roten und des Blauen (Quelle: Pfläging)

Komplexität im Management ist seit der digitalen Transformation wieder in den Fokus gerückt. Wie können Unternehmen mit wachsender Komplexität und Dynamik von Märkten umgehen? Wie kann zeitgemäße Führung und Struktur in Unternehmen etabliert werden und wie können wir mithilfe von speziellen Methoden Komplexität reduzieren? Laut dem Autor Niels Pfläging sollen Unternehmensstrukturen genauso lebendig wie Märkte sein.

Es verändert sich aktuell viel in unserer Welt durch den digitalen Wandel. Die schnelle und massive Einführung von Technologie geht nicht spurlos an uns vorbei. So ist einerseits unsere Arbeit davon betroffen. Ohne Computer und Internet steht die Arbeit heute in vielen Betrieben still. Handwerker fertigen mit digitaler Hilfe Möbel oder Autoteile, Architekten erstellen in Windeseile Baupläne, wo sie vorher aufwendige Zeichnungen anfertigen mussten. Mitarbeiter sind über die sozialen Netzwerke miteinander verbunden, digitale Programme erleichtern Arbeitsabläufe und Absprachen untereinander. Arbeitnehmer können sich auf wesentliche Aufgaben konzentrieren und sind beispielsweise nicht mehr 
mit Verwaltungsaufgaben überlastet (Quelle: Bildungsexperten).

Der digitale Wandel ist also mehr als Arbeiten mit Internetanschluss. Er verändert mit einer rasanten und unaufhaltsamen Geschwindigkeit auch Unternehmensstrukturen. Wo einst in Organisationen mit Kontrollgremien und Managern noch oft analog kommuniziert worden ist, finden sich mittlerweile vernetzte und projektorientierte Unternehmen, welche auf Basis von Technologie miteinander kommunizieren. Agile Methoden werden zunehmend zum State of the Art in Unternehmen. Es gilt also auch die Organisation an die Menschen und die neuen Rahmenbedingungen anzupassen.

Wirklich gute Führung war schon immer eine Herausforderung. Das war schon vor dem digitalen Wandel so. Durch die Digitalisierung kommen neue Anforderungen hinzu: bedingungslose Offenheit, maximale Beweglichkeit, sinnvolle Vernetzung und hohe Partizipation. Dies fasst der Begriff Digital Leadership zusammen. So ist es nun u. a. Aufgabe von Führungskräften Werte vorzuleben, zu fördern und zu vermitteln.

Mit allen diesen Themen befasst sich dieses Buch und soll Ihnen einen Überblick über die Veränderungen geben, welche der digitale Wandel mit sich bringt. Ob nun nur gewisse Stellschrauben im Unternehmen verändert werden oder ob es fundamentaler wird, ist aktuell nicht klar, aber eines ist auf jeden Fall klar: Es wird sich etwas ändern, und so wie Petry in seinem Buch zu Digital Leadership nutze ich auch gern das chinesische Sprichwort "Wenn der Wind der Veränderung bläst, bauen die einen Schutzmauern und die anderen Windmühlen" für den digitalen Wandel. 


\section{Quellen}

Pfläging, N., \& Hermann, S. (2015). Komplexithoden: Clevere Wege zur (Wieder)Belebung von Unternehmen und Arbeit in Komplexität. München: Redline Verlag.

http://www.bbrt.ch/files/Unternehmensfuehrung_im_Wissenszeitalter_ KMU_Magazin_10-08.pdf

https://blog.hubspot.de/marketing/technologie-digitales-zeitalter

https://www.bitkom.org/Themen/Digitale-Transformation-

Branchen/Digitale-Transformation/index.html

http://www.bildungsxperten.net/job-karriere/arbeit-im-digitalen-

wandel-chancen-und-risike 


\section{Kapitel 1 \\ DIGITALER WANDEL}

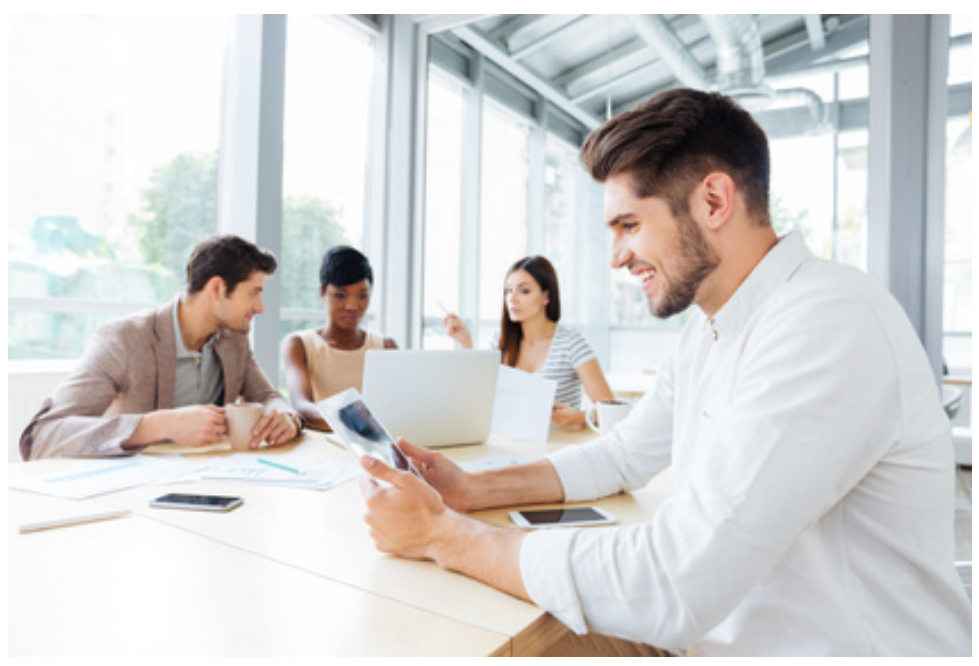

Wie oft schauen Sie pro Tag auf Ihr Smartphone, ohne dass es klingelt? Selbst während der Arbeitszeit ertappen wir uns dabei, unser Display regelmäßig auf neue Nachrichten zu checken, ob es nun blinkt oder nicht. Aber warum empfinden wir dieses Bedürfnis, das Telefon in die Hand zu nehmen, ohne dass wir angerufen werden oder eine Nachricht erhalten haben? (Quelle: Hubspot) Der digitale Wandel ist schon seit einiger Zeit in vollem Gang und trifft alle Bereiche, nicht nur Unternehmen.

In diesem Kapitel zeige ich zuerst auf, was der digitale Wandel ist, dann welche Herausforderungen er für Unternehmen bietet und am Ende zeigt ein Dialog mit Boris Gloger auf, wie man diesem Wandel begegnen kann. 


\section{WAS IST EIGENTLICH DER DIGITA- LE WANDEL? - DEFINITION}

Originalartikel: https://agile-unternehmen.de/was-ist-digitaletransformation-definition/

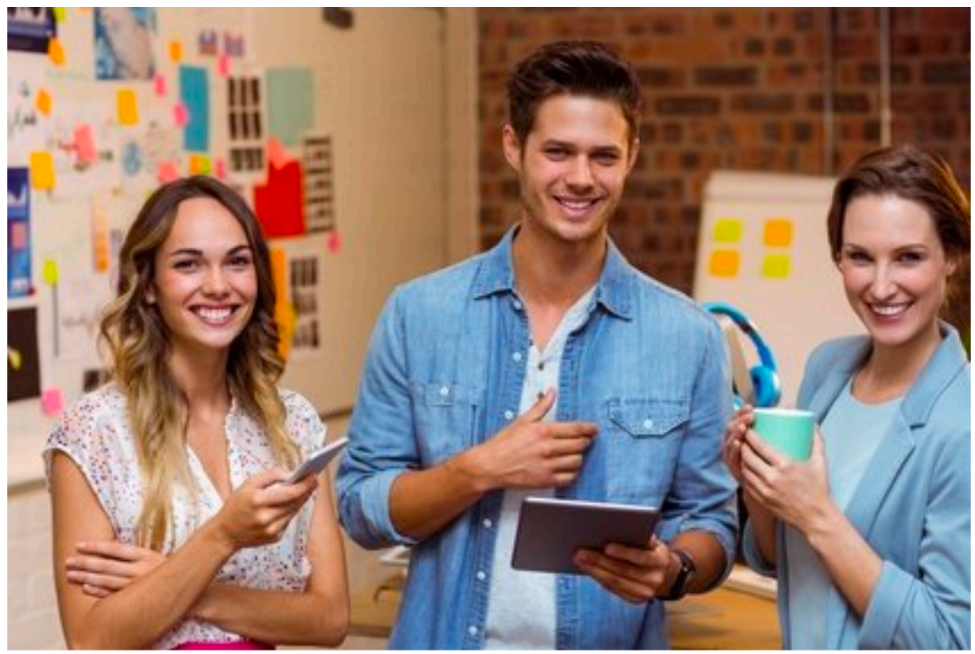

Kein Wort wird aktuell so oft in den führenden Magazinen verwendet wie die digitale Transformation. So stellt sich also die Frage: Was ist die digitale Transformation und was ist die Definition? Der Artikel zeigt neben einer formalen Definition auch Gründe, Potenziale und Akteure des „Buzzwords“.

\section{DIGITALE TRANSFORMATION - DEFINITION}

Die digitale Transformation bezeichnet einen fortlaufenden, in digitalen Technologien begründeten Veränderungsprozess, der die gesamte Gesellschaft und 


\section{DIGITALER WANDEL}

insbesondere Unternehmen betrifft. Basis der digitalen Transformation sind digitale Technologien, die in einer immer schneller werdenden Folge entwickelt werden und somit den Weg für wieder neue digitale Technologien ebnen (Wolan). Die digitale Transformation bezeichnet also Änderungsprozesse aufgrund von digitalen Technologien in hoher Geschwindigkeit. Als treibende Kraft dieser Digitalisierung werden in vielen Quellen die Veränderungen der Kundenwünsche genannt, welche mit digitalen Technologien anders bedient werden können. Ein Beispiel hierfür ist der Streamingdienst Spotify, welcher Musik immer, überall, jederzeit und kostengünstig zugänglich macht.

Insgesamt unterscheidet die Literatur zwischen Enablern und Akteuren. Enabler bedeutet so viel wie „Ermöglicher" und schließt u. a. Folgendes ein:

- zum Ersten digitale Technologien (Cloud Computing, ...),

- digitale Infrastrukturen (Protokolle, ...) und

- zu guter Letzt digitale Anwendungen (OnlineBanking, ...).

Auf Basis dieser „Ermöglicher“ entstehen neue Verwertungspotenziale in neuen Bereichen. Unternehmen beginnen diese zu erkunden und in ersten Pilotprojekten auszuprobieren. Beispiele sind digitale Geschäftsmodelle und digitale Wertschöpfungsnetzwerke. Digitale Geschäftsmodelle beschreiben die Veränderung von Geschäftsmodellen, welche vorher analog abliefen, zu einem digitalen Modell. Ein Beispiel ist das OnlineTicket der deutschen Bahn. Digitale Wertschöpfungsnetzwerke sind z. B. virtuelle Teams. 


\section{DIGITALER WANDEL}

Nun kommen wir zu den Akteuren des digitalen Wandels. In erster Linie trifft der Wandel Menschen: Unternehmer, Forscher und Mitarbeiter sowie Bürger von Deutschland. Diese können nun in Echtzeit miteinander kommunizieren oder ihr Leben im Live-Stream mit anderen teilen. Erst gestern habe ich mich mit meiner Zahnbürste vernetzt. Diese neuen Möglichkeiten haben jedoch auch Schattenseiten: Mangelnder Datenschutz und Information Overload sind die Folgen.

Ein anderer Akteur sind Unternehmen. Hier wandelt sich die Arbeit in eine sogenannte digitale Arbeit und Geschäftsmodelle wandeln sich fundamental. Auch betroffen sind Wissenschaft und Lehre in Form neuer Möglichkeiten zur Archivierung, Publikation und Aufbereitung der Forschung sowie E-Learning. Auch bietet die Digitalisierung zahlreiche Herausforderungen für Regierungen. Hauptaufgabe wird die Lenkung dieser Transformation durch Gesetze und Richtlinien sowie die eigene Transformation der behördlichen Abläufe sein. 


\section{DIGITALER WANDEL}

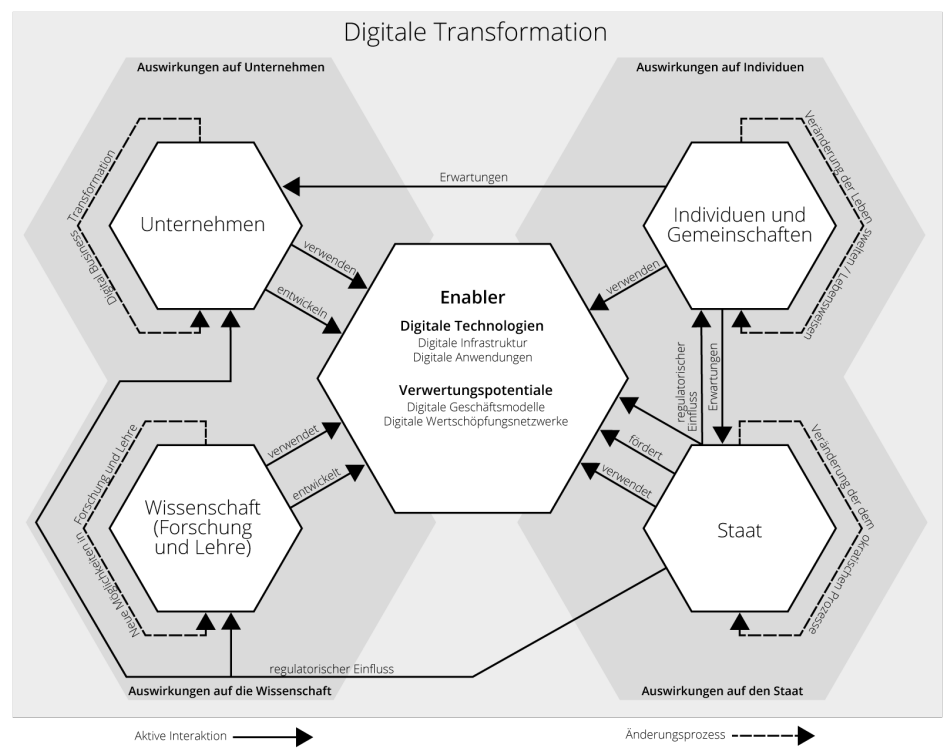

Einfluss und Auswirkungen des Megatrends auf Forschung, Gemeinschaft, Staat und Forschung (von Thomas Kofler-eigenes Werk, CCBY-SA 4.0, https:/(commons.wikimedia.org/w/index.php?curid=48399771)

\section{TREIBER DER DIGITALEN TRANSFORMATION}

Doch warum sprechen wir ausgerechnet jetzt von einer digitalen Transformation? Warum ist die Geschwindigkeit nun schnell genug, dass wir darüber so intensiv forschen? Zur Antwort dieser Frage hat Petry (2016) das folgende Diagramm geliefert und Treiber für den digitalen Wandel geliefert. Zu Beginn steht das exponentielle Wachstum von Unternehmen mit den Kernthemen der Vernetzung, Al und Big Data. Konsequenzen sind die Veränderung von Arbeit, Kundenverhalten und Wettbewerbssituation. 


\section{DIGITALER WANDEL}

Treiber

Kernthemen

Einfluss
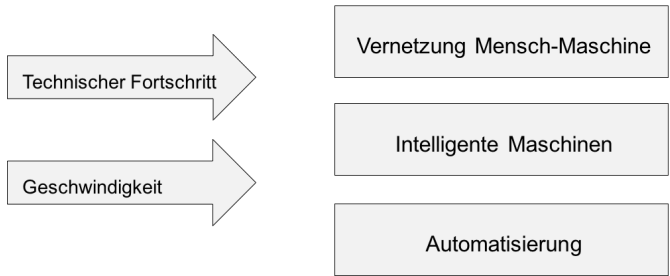

Neue Kundenwünsche

Neue Märkte

- Neue Produkte

- Neue Geschäftsmodelle

- VUCA-Welt

- Arbeit 4.0

Treiber und Konsequenzen der digitalen Transformation (eigene Darstellung nach der Idee von Petry 2016).

\section{ABER WIR DIGITALISIEREN UNS DOCH SCHON SEIT 50 JAHREN!}

Die größte aktuelle Kritik an der These zur digitalen Transformation ist, dass wir uns bereits seit vielen Jahren digitalisieren. Das ist richtig und trifft vollkommen den Kern der Kritik. Schaut man in aktuelle Literatur oder in Unternehmen, dann bemerkt man jedoch einen gravierenden Unterschied.

Während wir vor vielen Jahren noch weitgehend an der Oberfläche eine Digitalisierung betrieben haben, bewegen sich Unternehmen nun in eine tiefgreifende Digitalisierung vor. Ein gutes Beispiel ist der Friseurbesuch. Hier buche ich über eine App meinen Termin. Dies ist definitiv digital. Jedoch wird der eigentliche Schneidevorgang noch analog ausgeführt. Klassisches People Business ist jedoch nicht skalierbar, da pro Kunde ein Mitarbeiter benötigt wird, ähnlich wie im Consulting, in welchem viele Tools den Berater unterstützen, aber die eigentliche Beratung noch analog stattfindet und der Berater selbst noch nicht digitalisiert ist. Mehr dazu im Artikel zu Consulting 4.0. Dies zeigt nur zwei Beispiele. 
Natürlich gibt es noch keine direkten Antworten auf diese Fragen, aber aktuell zeigt sich der Trend zu einer tiefgreifenden Digitalisierung mit fundamentaler Verhaltensänderung. Digitale Transformation ist also eine tiefgreifende soziale Änderung im Verhalten durch digitale Technologien. Ein existierendes Beispiel ist der Paradigmawechsel von Remote-Arbeit statt Anwesenheitspflicht.

\section{EVOLUTIONSSTUFEN DER DIGITALEN TRANSFORMA- TION}

Im Blog der Social Collaboration Academy habe ich außerdem eine Antwort auf die Frage, ob wir uns schon ewig digitalisieren, gefunden. Es gibt nämlich laut diesem Artikel vier Stufen der Digitalisierung.

- Die erste Evolutionsstufe dauerte von ca. 1990 bis 2000 und beschäftigte sich mit der Vernetzung von Computern.

- In der zweiten Evolutionsstufe von ca. 2000 bis ca. 2015 ging es primär um eine allgemeine Akzeptanz und allgemeine alltägliche Einführung und Nutzung mobiler Geräte.

- Die jetzt vor uns liegende dritte Evolutionsstufe, deren Dauer Experten von ca. 2015 bis 2030 veranschlagen, hat Themen wie allgemeine Reife der Systeme und das Internet der Dinge im Fokus.

- Mit der vierten Evolutionsstufe ab ca. 2030 soll es zu einer vollkommenen Verschmelzung der realen und digital vernetzten Welt kommen. 
$1990-2000$

Computer im Alltag

$2000-2015$

Mobile Geräte im Alltag

$2015-2030$

Intelligente mobile Geräte im Alltag

\begin{abstract}
Ab 2030
Vernetzungsgesellschaft

(reale Welt $=$ digitale Welt $)$

Die Evolution der digitalen Transformation (eigene Darstellung nach einer Idee der Social Academy)
\end{abstract}

\title{
TRANSFORMATION, WANDEL, EVOLUTION ODER RE- VOLUTION?
}

Es finden sich viele Begriffe für die digitale Transformation und viele Meinungen. Ist es nun eine digitale Transformation? Eine Transformation hat einen Anfang und ein Ende. Wandel steht für einen Zustand, der in einen anderen Zustand übergeht. Evolution steht für die friedliche Weiterentwicklung von etwas und Revolution für eine grundlegende, oft gewaltsame Neuerung. Lesen Sie dazu auch einen Artikel zur digitalen R(evolution). Viele sagen auch einfach Digitalisierung. Dies würde einfach bedeuten: „Etwas wird eben digital“. Alle Begriffe könnten zutreffen. 


\section{WAS IST DIGITALE TRANSFORMATION?}

Viele Unternehmen treiben schon lange die Digitalisierung der Gesellschaft. Von der digitalen Transformation sprechen wir nun aber wegen der Geschwindigkeit und der tiefgreifenden digitalen Änderungen. Wurden vorher noch oft „an der Oberfläche“ digitale Technologien verwendet, sind es nun tiefgreifende Änderungen bis ins Geschäftsmodell. Es bleibt spannend, wo die digitale Reise hingeht.

\section{Quellen}

Michael Wolan: Digitale Innovation: Schneller. Wirtschaftlicher. Nachhaltiger. BusinessVillage, 2013.

Thorsten Petry: Digital Leadership. Haufe, 2016.

http://socialcollaborationacademy.com/digitalisierung/das-digitalezeitalter/00108/ 


\section{DIGITALISIERUNG IM MITTEL- STAND TRIFFT AUF TRADITION}

Originalartikel: https://agile-unternehmen.de/digitalisierung-immittelstand-tradition/

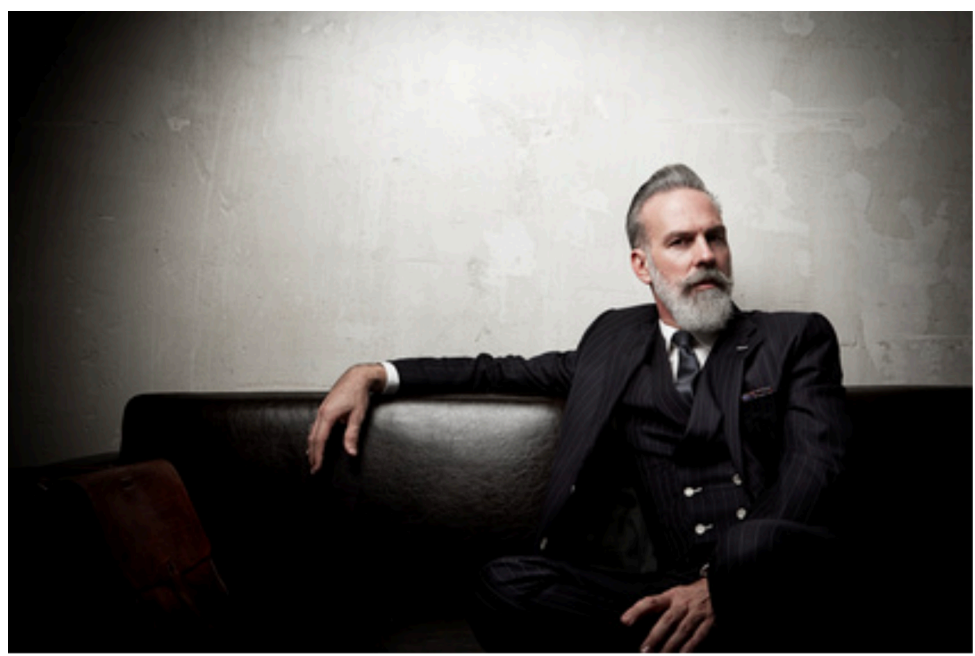

Die Digitalisierung aller Lebensbereiche hat zu steigender Komplexität geführt und Unternehmen finden sich durch diese Entwicklung in einem Zwiespalt wieder. Ein Beispiel ist die mittelständische Unternehmensberatung, welche eine der Branchen ist, die exzellent von der Digitalisierung profitiert, allerdings primär als diejenige, die die Vielzahl der Projekte zur Digitalisierung von Unternehmen verschiedenster Branchen aufsetzt, steuert und durchführt. Jedoch ist diese oft selbst kaum digitalisiert. Mehr Informationen können Sie meinem Artikel zu Consulting 4.0 entnehmen. 


\section{DIGITALER WANDEL}

Das Wettrennen um die digitale Vorherrschaft ist durch ein hohes Veränderungstempo gekennzeichnet. Insbesondere amerikanische und asiatische Unternehmen befinden sich auf der Überholspur und drängen weltweit Wettbewerber aus den Märkten. Doch wie sieht es in Deutschland aus? Wie schreitet die digitale Transformation im deutschen Mittelstand voran?

\section{DIE TRADITION IM MITTELSTAND}

Den digitalen Wandel erfolgreich zu vollziehen, ist derzeit Aufgabe aller Unternehmen und Branchen. In Deutschland wird das Wirtschaftswachstum vor allem durch den Mittelstand forciert. Als Rückgrat der deutschen Wirtschaft zählen besonders Traditionsunternehmen aufgrund ihrer Innovationsfähigkeit und der Technologiekompetenzen als eine wichtige Stütze. Laut einer Studie des BMWI wird $57 \%$ der Wirtschaftsleistung durch den deutschen Mittelstand erbracht.

Mittelständische Unternehmen haben eine lange Tradition und sind oft durch eine langfristige Orientierung geprägt. Dank einer hohen Wertschöpfungstiefe und dem Fokus auf die Qualität ihrer innovativen Produkte bieten sich viele Möglichkeiten der Prozessautomatisierung und der Aufwertung von Produkten mit digitalen bzw. softwarebasierten Modulen. Wirft man einen Blick auf die aktuelle digitale Studie der KfW, findet man Sätze wie:

- Nur ein Fünftel der mittelständischen Unternehmen hat die digitale Vernetzung von Produkten und Dienstleistungen begonnen und kann damit zu „Vorreitern“ gezählt werden. 


\section{DIGITALER WANDEL}

- Der Ausbau der Digitalisierung geht in den meisten Unternehmen voran, aber überwiegend in kleinen Schritten.

- Defizite bei IT-Kompetenzen, Datensicherheit und Internetgeschwindigkeit erschweren die Digitalisierung im Mittelstand.

- Fokusthema Industrie 4.0: Projekte sind typische Innovationsprojekte; Qualifikationsanforderungen ändern sich, Geschäftsmodelle nur graduell.

Jean Jaurés prägte einst den wunderbaren Ausspruch: "Tradition heißt nicht, Asche verwahren, sondern eine Flamme am Brennen halten." Fast kein Traditionsunternehmen würde wohl heute noch existieren, wenn es sich im Laufe ihrer Geschichte nicht immer wieder an den eigenen und bewährten Grundsätzen sowie Traditionen orientiert hätte. Aber reicht der Verweis auf die Vergangenheit im modernen Wettbewerb aus, um langfristig konkurrenzfähig zu bleiben? Denn wie ich bereits im Artikel zur digitalen R(evolution) sagte: Wenn der Wind der Veränderung bläst, bauen die einen Schutzmauern und die anderen Windmühlen.

\section{DIGITALISIERUNG IM MITTELSTAND}

Der digitale Wandel betrifft jeden: Arbeitgeber wie Arbeitnehmer, Studierende und Familien, junge und alte Menschen. Auf dem Land und in der Stadt. In der Werkstatt nebenan wie im Großbetrieb. So ist auch Digitalisierung im Mittelstand ein Thema. Für diesen ist es an der Zeit, seine Grundwerte, also seinen Fokus auf nachhaltiges Unternehmenswachstum, langfristige und enge Kunden- und 


\section{DIGITALER WANDEL}

Lieferantenbeziehungen sowie die Zufriedenheit seiner Mitarbeiter, erfolgsversprechend in das digitale Zeitalter zu überführen. So gilt es den Zielkonflikt zwischen der langfristigen Strategie der Traditionsunternehmen und der hohen Geschwindigkeit von Innovationen und Veränderungsprozessen der Digitalisierung zu lösen. Nun werden sich sicherlich nicht alle Bereiche fundamental ändern und jeder Mitarbeiter zu $100 \%$ eigenverantwortlich arbeiten, aber Unternehmen sollten auf diese Werte zusteuern und zumindest etwas mehr Selbststeuerung als gewohnt zulassen. Einen Ansatz für eine solche Veränderung der Werte liefert Brandes in der folgenden Abbildung, welche ich im Kontext der Digitalisierung im Mittelstand interpretiere. 

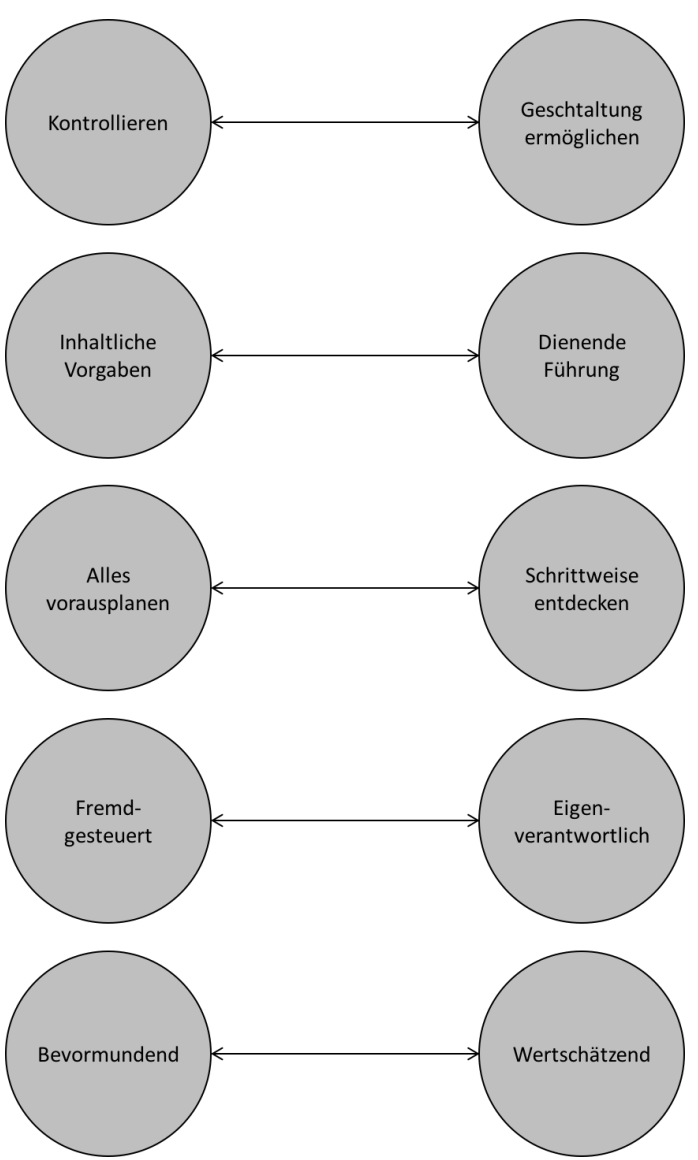

Es ist nicht wichtig, alle Bereiche neu zu erfinden, sondern nur gewisse Stellschrauben feiner zu justieren (eigene Darstellung angelehnt an die Idee von Brandes)

\section{TRADITION TRIFFT DIGITALISIERUNG}

Die digitale Transformation ist Unternehmen angekommen. Besonders der deutsche Mittelstand ist ein wichtiges Rückgrat der deutschen Wirtschaft. Im Vergleich zu Startups verfügt dieser jedoch oft über eine lange Tradition, 


\section{DIGITALER WANDEL}

welche nicht einfach zu verändern ist. Jedoch wird sich nicht jeder Bereich fundamental verändern. Aus diesem Grund wird eine Steigerung von Agilität in wichtigen Kernbereichen empfohlen. Grundsätzlich gilt also für Traditionsunternehmen, ihre Grundwerte, also ihren Fokus auf nachhaltiges Unternehmenswachstum, langfristige und enge Kunden- und Lieferantenbeziehungen sowie die Zufriedenheit ihrer Mitarbeiter, erfolgsversprechend in das digitale Zeitalter zu überführen. Ein Ansatz wurde hier im Artikel vorgestellt. Klar ist jedoch: Aktuelle klassische Methoden von Traditionsunternehmen sind oft nicht mehr optimal im Kontext der digitalen Transformation und sollten neu bewertet werden. Die folgende Abbildung zeigt, wieso gewisse Grundwerte sich ändern müssen. Dies führt dazu, dass sich der Fokus von Maschinen und Planung hin zu Kreativität und Menschen verschiebt. 


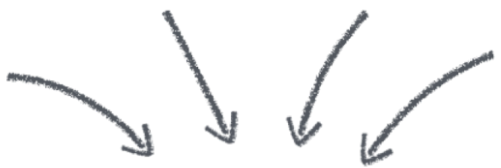

Machine

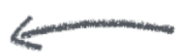

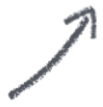
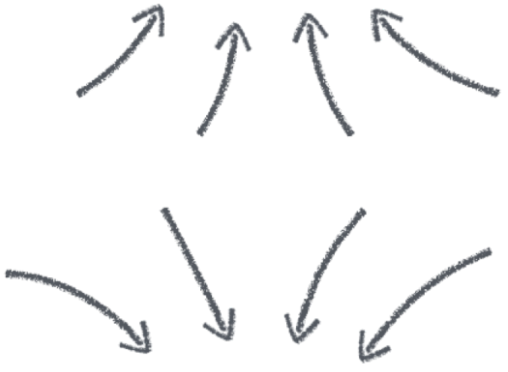

$\rightarrow$ Mensch
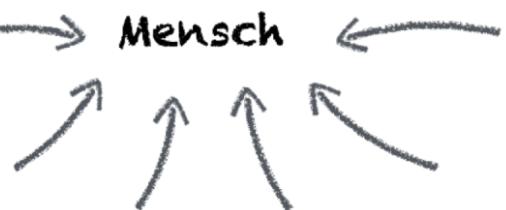

Während vorher Maschinen die Wirtschaft bestimmt haben, rückt nun der Mensch immer mehr in den Mittelpunkt der Digitalisierung im Mittelstand (eigene Darstellung)

\section{QUELLEN}

Brandes, U., Gemmer, P., Koschek, H., \& Schültken, L. (2014). Management Y. München: Campus Verlag.

https://www.kfw.de/PDF/Download-Center/Konzernthemen/Research/PDFDokumente-Studien-und-Materialien/Digitalisierung-im-Mittelstand.pdf

https://www.bmwi.de/BMWi/Redaktion/PDF/W/wirtschaftsmotormittelstand-zahlen-und-fakten-zu-den-deutschenkmu,property=pdf,bereich=bmwi2012,sprache=de, $\mathrm{wb}=$ =true.pdf http://www.bmwi.de/Redaktion/DE/Publikationen/Mittelstand/wirtschafts motor-mittelstand-zahlen-und-fakten-zu-den-deutschen-kmu.html

https://www.kfw.de/PDF/Download-Center/Konzernthemen/Research/PDFDokumente-Studien-und-Materialien/Digitalisierung-im-Mittelstand.pdf 


\section{DIALOG MIT BORIS GLOGER ZU SEINEM NEUEN BUCH „SCRUM - THINK B!G“}

Originalartikel: https://agile-unternehmen.de/boris-gloger-scrumthink-big/

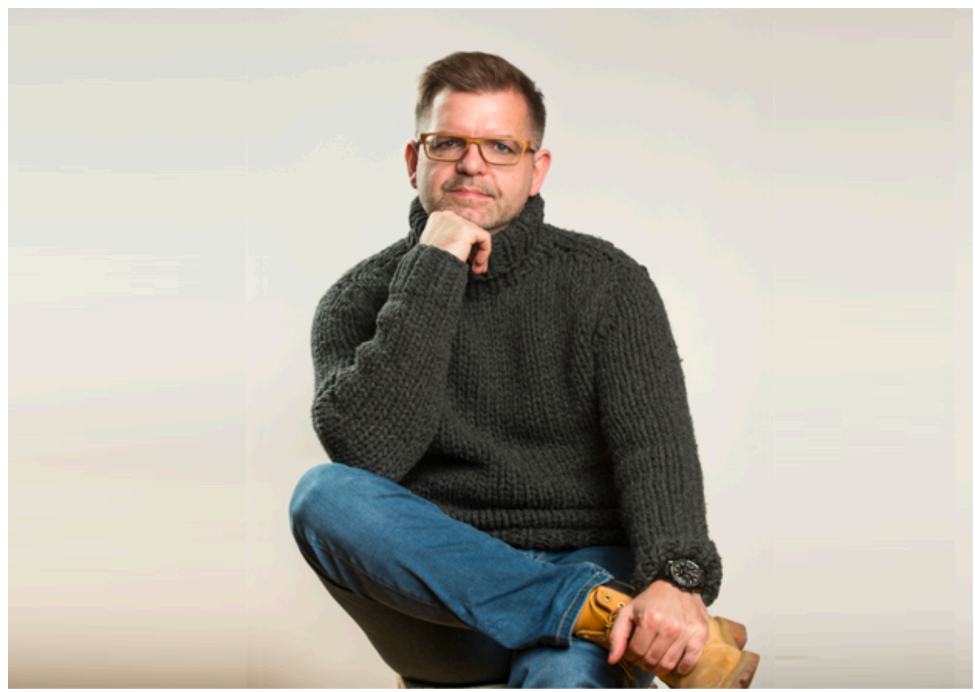

Boris Gloger ist Gründer und Geschäftsführer der borisgloger consulting gmbh (Baden-Baden, Wien). In seinen Büchern zeigt er das breite Anwendungsspektrum von Scrum - von der Produktentwicklung bis zum Management großer Organisationen. Als weltweit erster von Ken Schwaber ausgebildeter Certified Scrum Trainer hat er wesentlich dazu beigetragen, dass sich Scrum in Europa, Südafrika und Brasilien als Standard der agilen Softwareentwicklung durchgesetzt hat. Seine Erfahrungen aus der 


\section{DIGITALER WANDEL}

Praxis hat er bereits in mehreren Büchern erfolgreich publiziert.

Agil ist Boris Gloger nicht nur in seinen Büchern, sondern auch in seiner täglichen Arbeit. Sein neues Buch "Scrum Think b!g“ erschien Mitte Februar. Das Besondere an diesem Buch ist, dass er es in kurzen Iterationen gemeinsam mit Lesern evaluiert und deren Feedback früh integriert hat. Im Zuge dieser Feedbackzyklen habe ich mich mit Boris Gloger getroffen und wir haben uns gemeinsam gewissen Spannungsfeldern der Skalierung von Scrum und der digitalen Transformation genähert. Über unsere Erkenntnisse lesen Sie im folgenden Artikel.

\section{FUNKTIONIERT SCRUM IN GROSSEN PROJEKTEN}

Der Grundsatz von Agilität ist Zusammenarbeit. Boris hat sich in seinem neuen Buch daher gefragt: „Welche Voraussetzungen müssen für die Zusammenarbeit eigentlich geschaffen werden?" Vor allem bei verteilten Teams und bei besonders komplexen Produkten zeigt sich, dass klassische Methoden schlichtweg nicht dafür geeignet sind, stellt Boris fest.

Und so passiert es, dass Konzerne große Software-Rollouts vollziehen und anschließend schnell bemerken, dass die Software nicht optimal an die Anforderungen der Nutzer angepasst wurde - weil nicht nur die Kommunikation mit den potenziellen Usern, sondern auch zwischen den beteiligten Teams nicht funktioniert hat. „Agile Methoden sind ein Ansatz! Ich habe bei meiner Arbeit immer wieder die Erfahrung gemacht, dass nach agilen Prinzipien gemanag- 
te Großprojekte zu deutlich besseren Ergebnissen führen." Hinter Scrum steckt allerdings mehr als nur ein paar wenige Prinzipien. Somit hat sich laut Boris herausgestellt, dass agile Rahmenwerke wie SAFe®, LeSS und Co. sehr kompliziert sind. „Scrum soll jedoch einfach sein und mit wenigen Schritten auskommen“, sagt Boris.

\section{DIE DIGITALE TRANSFORMATION MACHT ALLE UN- TERNEHMEN ZU SOFTWAREUNTERNEHMEN!}

Die digitale Transformation ist in aller Munde und wird natürlich auch in "Scrum - Think b!g“ thematisiert. Logischerweise war die IT als Erstes von der Digitalisierung betroffen. „Digitalisierung ist der Treiber von Agilität“, so Boris, "daher ist es notwendig, schneller auf Veränderungen zu reagieren als bisher." In der Softwareentwicklung sei das schon ganz gut gelungen, stellt Boris fest. Scrum hat aus seiner Sicht die Softwareentwicklung grundlegend verändert und IT-Teams darauf vorbereitet, schnell und flexibel auf äußere Anforderungen zu reagieren. Doch nun verlässt die Digitalisierung die IT und betrifft mehr oder weniger alle Bereiche und Branchen. Jetzt scheint also die richtige Zeit gekommen zu sein, um über die Rolle der Skalierung von Scrum im eigenen Unternehmen nachzudenken.

"Ich möchte nicht noch ein Buch über den Prozess von Scrum schreiben, sondern Unternehmen helfen, die richtigen Rahmenbedingungen für Scrum zu schaffen." (Boris Gloger) 


\section{DAS MANAGEMENT IN DER KRISE! BRAUCHT MAN ES NOCH IM SKALIERTEN SCRUM?}

„Viele bekannte Agilisten waren selbst nie Topmanager in großen Unternehmen und haben daher auch keine Projekte im Wert von mehreren hundert Millionen Euro verantwortet. Auch ich zähle dazu und deswegen möchte ich keine direkten Handlungsempfehlungen, sondern Werte und Mechanismen an die Manager weitergeben." Die Werkzeuge des klassischen Scrum, die in kleinen Teams funktionieren, sind oft kaum auf das Zusammenwirken von mehreren Teams übertragbar. Was jedoch laut Boris übertragbar ist, sind Mechanismen der Selbstorganisation. Er beschreibt dies mit der Theorie, dass ein Team eine Selbstreferenz (Selbstbetrachtung) benötigt, zum Beispiel in Form von Retrospektiven, Dailys und Retros. Boris unterstreicht diesen Ansatz anhand des Beispiels von Daimler, wo aktuell mithilfe von Werten und Prinzipien ein Paradigmenwechsel angestrebt wird. „Dieter Zetsche nutzt gezielt die Medien, um den Mitarbeitern seine Werte zu vermitteln und seine Ziele rund um die digitale Transformation zu erklären." Aus Sicht von Boris ist es wichtig, Mechanismen wie Partizipation und die Lust an der Arbeit zu etablieren, damit ein agiles Mindset wachsen kann. Die Aufgabe der Führung ist es, dazu einzuladen und Agilität möglich zu machen.

\section{„SCRUM IN DER HARDWARE UND AUSSERHALB DER IT. SOWAS GEHT DOCH NICHT!“}

Wenn man Scrum skaliert, muss man zwangsläufig über den Rand der IT hinausschauen. Speziell die Produktentwicklung, etwa von Hardware, setzt die Zusammenarbeit 


\section{DIGITALER WANDEL}

von Spezialisten unterschiedlichster Domänen voraus. Als er sich mit der Entstehung von Scrum beschäftigt hat, hat Boris festgestellt, dass der Ursprung der Agilität eigentlich in der Hardwareentwicklung liegt. Hardwareprojekte waren schon immer von verschiedenen Keyplayern aus unterschiedlichen Domänen abhängig und die Herausforderung bestand immer darin, diese zum gemeinsamen Nachdenken anzuregen. Unterstützt durch eigene Prozesse wurde effiziente Zusammenarbeit ermöglicht, mit dem Ziel, ein gutes Projekt auf den Markt zu bringen. Boris bringt in diesem Zusammenhang ein gutes Beispiel: „Die NASA war in den 1960ern eigentlich wahnsinnig agil. Sie hatte für das Raumfahrtprojekt knapp 300 Teams bereitgestellt. Jedes Team erhielt ein Radio mit verschiedenen Kanälen und einem Public-Kanal. Damit war schon damals eine recht agile Kommunikation möglich." Als zweites Beispiel führt Boris den US-General Stanley McChrystal an, der von 2003 bis 2008 Kommandeur des Joint Special Operations Command (JSOC) im Irak und in Afghanistan war. „Bei den Dailys mussten jeden Tag alle 7000 Soldaten seiner Einheit über Videokonferenzsysteme mitmachen. Begründet hat er das damit, dass seine Entscheidungen bei kritischen Einsätzen zu langsam an die Front gelangen würden. Wenn ein Elitesoldat bei Planänderungen erst den General fragen muss, funktioniert das im Gefecht wohl kaum." Im Dialog stellten wir fest, dass solche Special Forces in ihrem Mindset wahrscheinlich nicht vollkommen agil sind, dass die Arbeit dieser Elitesoldaten aber absolut agil und effizient ist. Und so gibt es aus Sicht von Boris zahlreiche weitere Beispiele in vielen Bereichen, die uns zeigen, dass Agilität nicht nur funktioniert, sondern sehr oft auch die natürlichste und sinnvollste Vorgehensweise ist. 


\section{DIGITALER WANDEL}

Den Skeptikern empfiehlt er: „Probiert es aus, denn es kann funktionieren!“

\section{AUSBLICK I: ÜBERGREIFENDE KOMMUNIKATION UND AGILITÄT}

"Ich bin dankbar, dass es Scrum gibt, denn dadurch habe ich gelernt, anders zu arbeiten." (Boris Gloger)

Der Druck wird höher - auf die Politik, in den Unternehmen und sogar in den Familien und auf Einzelpersonen. Es wird also mehr Agilität verlangt, so Boris, und daher sei es absolut notwendig, endlich mehr miteinander zu reden. Obwohl sich draußen die Märkte laufend verändern und neue Anforderungen stellen, existieren in vielen Unternehmen noch immer Silos und Lager, die eher gegenstatt miteinander arbeiten. Boris beobachtet, dass Scrum zumindest in der IT die Fronten aufgeweicht hat: Tester und Entwickler reden miteinander und versuchen einander zu verstehen, auch Designer, Backend- und Frontendentwickler vernetzen sich. Boris wünscht sich, dass diese Entwicklung auf sämtliche Bereiche übergreift und die Menschen verschiedener Disziplinen mit einem gemeinsamen Ziel vor Augen wieder intensiver ins Gespräch kommen.

\section{AUSBLICK II: GESUNDER MENSCHENVERSTAND UND AGILITÄT}

Wenn Boris noch einen Wunsch frei hätte, dann diesen: Geben Sie Prozesse nicht auf, bis Sie die dahinterliegenden Prinzipien verstanden haben. „Nehmen wir eine Ampel als Beispiel. Diese soll den Verkehr regeln - das ist auch gut so! 


\section{DIGITALER WANDEL}

Wenn aber kein Auto da ist, warum soll ich dann warten? Warum kann ich nicht den Prozess missachten und bei Rot über die Ampel gehen?“ Was meint Boris damit? Das ist eigentlich ganz einfach: Prozesse sind weiterhin richtig und wichtig. Allerdings sollten sie nicht zur unumstößlichen Wahrheit erhoben werden, sondern Empfehlungen sein, die nicht immer strengstens befolgt werden müssen. Wir sollten einfach wieder öfter unserem gesunden Menschenverstand folgen.

\section{WIE SEHEN SIE DAS?}

Ich möchte Boris für den tollen Dialog und die spannenden Inhalte danken. Wenn Sie mehr über die Skalierung von Agilität erfahren möchten, schauen Sie doch mal in das Buch von Boris Gloger oder auf seine Website. 


\section{Kapitel 2 \\ Arbeit 4.0}

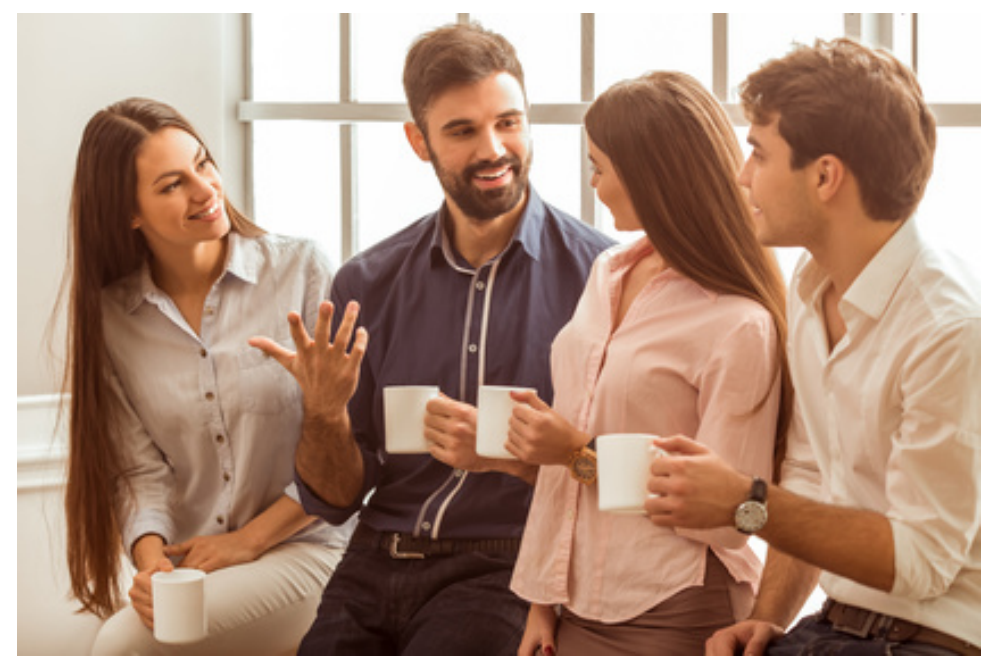

Es sind die gegenwärtigen Entwicklungen in Wirtschaft und Gesellschaft, die Unternehmen dazu zwingen, sich mit neuen Formen der Arbeit auseinanderzusetzen. Zahlreiche neuartige Modelle finden Einzug in den Köpfen deutscher Manager - Modelle, die oft bereits in anderen Unternehmen erprobt sind. Remote-Arbeit und Open Spaces sind zwei dieser Beispiele, die eine neue Art der Führung fördern.

In diesem Kapitel habe ich zuerst einen Artikel ausgesucht, der erklärt, was Arbeit 4.0 eigentlich ist und anschließend zeige ich den Einfluss der Digitalisierung auf die Arbeit 4.0 mit einem weiteren Artikel. Am Ende rundet der Dialog mit Dr. Andreas Zeuch zur neuen Arbeit das Kapitel ab. 


\title{
WAS IST ARBEIT 4.0? - DEFINITION
}

\author{
Originalartikel: https://agile-unternehmen.de/was-ist-arbeit-4-0- \\ definition/
}

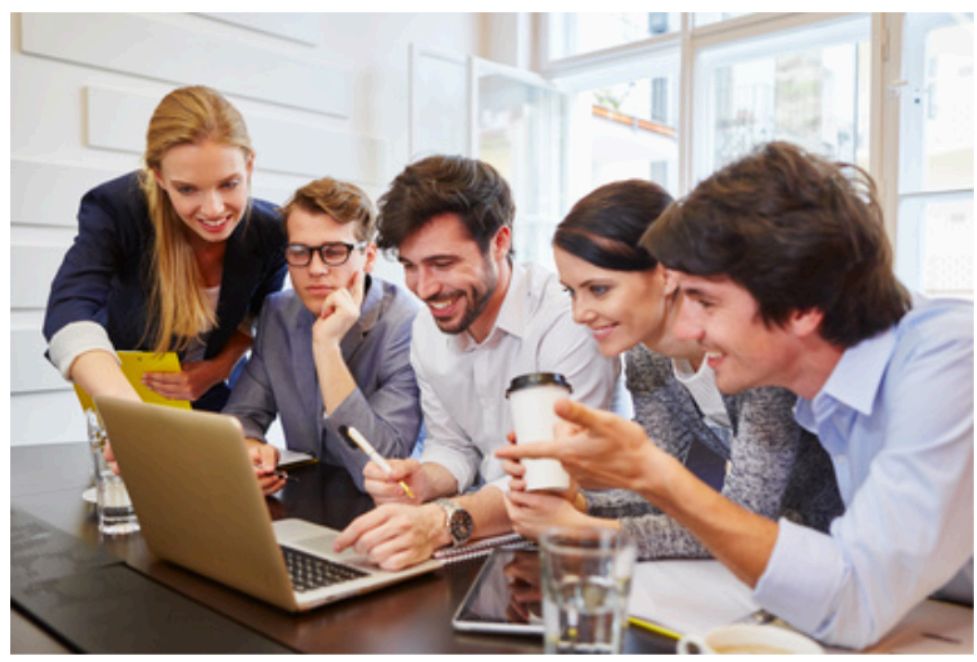

Die Zukunft der Arbeit im Rahmen der Digitalisierung vieler Lebensbereiche wird unter dem Begriff "Arbeit 4.0“ zusammengefasst. Klingt einfach, ist aber praktisch hochkompliziert: Hinter dem Schlagwort „Arbeit 4.0“ verbergen sich eine Fülle bislang ungeklärter arbeitsrechtlicher Fragen für Arbeitgeber, Führungskräfte und Mitarbeiter, so das Manager Magazin. Dieser Artikel soll deswegen die Idee und ausgewählte Konzepte hinter dem Begriff Arbeit 4.0 zusammenfassen. Die grundlegende Frage des Artikels lautet deswegen: Was ist Arbeit 4.0 und gibt es eine Definition? 


\section{ARBEIT 4.0}

WAS IST ARBEIT 4.0?

Als Begriff befasst sich „Arbeit 4.0“ mit der Zukunft der Arbeit im digitalen Zeitalter. Hintergrund sind vor allem die Probleme (aber auch Chancen), die eine fortschreitende Technisierung im Arbeitsmarkt und den generellen Strukturen von Unternehmen bewirkt. Im 3. Roundtable wurde darüber diskutiert, was der Begriff im Kontext der Teilnehmer bedeutet. Folgende Charakteristiken der Arbeit 4.0 wurden im Konsens verabschiedet:

- $\quad$ Arbeit 4.0 muss agil sein!

- Agilität braucht Freiraum!

- Gute Arbeit basiert auf Freiwilligkeit!

- Der Begriff der „Führung“ muss einem „Anleiten“ weichen!

- $\quad$ Arbeit wird künftig dynamischer und kurzzyklischer!

- $\quad$ Arbeit 4.0 wird digital unterstützt! 

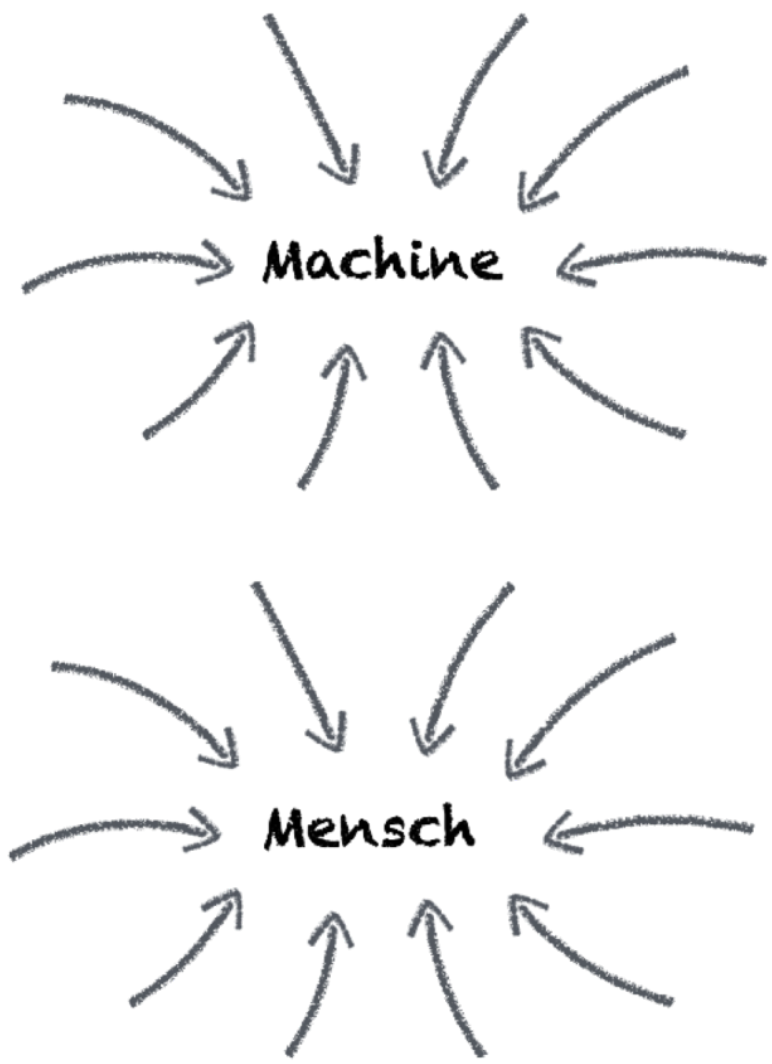

Fokus der Arbeit 4.0 liegt nicht mehr auf Maschinen, sondern auf dem Menschen (eigene Darstellung)

Im Kontext der Arbeit 4.0 steht also nicht mehr wie vor vielen Jahren noch technische Expertise, sondern der Mensch im Mittelpunkt. Sätze wie Technologie als Helfer des Menschen und der Mensch als "Überwacher" der Technologie unterstreichen diese These. Insgesamt haben die Teilnehmer in meinem Roundtable zur Arbeit 4.0 drei Ansätze vorgestellt, welche die Arbeit 4.0 ausgestalten könnten. 


\section{DER DIGITALE ARBEITSPLATZ}

Ein Enabler der Arbeit 4.0 ist laut vielen Magazinen die schnelle und fortschreitende Digitalisierung. So wird der Arbeitsplatz zunehmend durch Tools und weitere Technologien unterstützt und somit „irgendwie digital“. Durch diese Veränderungen ergeben sich neue Möglichkeiten wie mobile oder Remote-Arbeit sowie eine hohe Vernetzung von Mitarbeitern. In einem Roundtable sagte ein Teilnehmer dazu: Arbeit ist nun bis zu 3 Tage pro Woche vom Home-Office aus möglich, ohne den Bezug zum Team zu verlieren.

Doch es scheint, dass diese Entwicklung noch am Anfang steht. In der Studie der WiWo zur Digitalisierung am Arbeitsplatz zeigt sich: „Die Digitalisierung ist daheim bereits weiter fortgeschritten als am Arbeitsplatz im Büro - das zeigt eine Studie unter deutschen Büroangestellten." Somit scheint als das volle Potenzial des digitalen Arbeitsplatzes noch nicht abrufbar zu sein. Lesen Sie mehr dazu im folgenden Artikel zum digitalen Arbeitsplatz. 

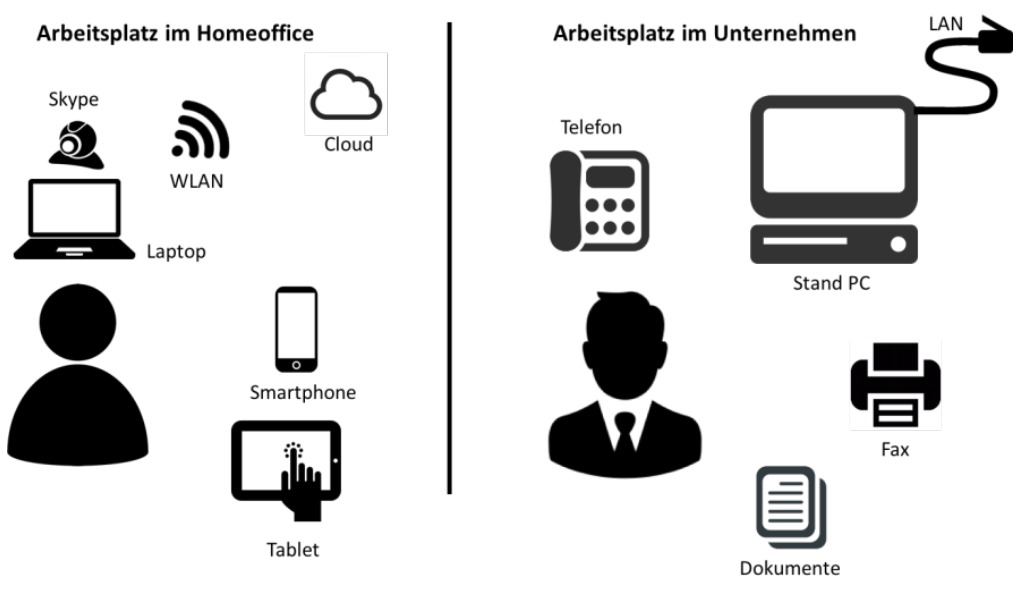

Ausstattung des Home Office vs. Büro (Quelle: eigene Darstel-

lung)

\section{NEW WORK - DEFINITION ARBEIT 4.0?}

Das Konzept New Work wurde vom austroamerikanischen Sozialphilosophen Frithjof Bergmann entwickelt, ausgehend von seiner Untersuchung des Freiheitsbegriffes. Der NewworkBlog sagt Folgendes: Der klassische „Nine-to-Five-Job“ im Büro wird schon bald der Vergangenheit angehören. Die Bindung an feste Arbeitsorte sowie standardisierte Zeiten und Organisationsstrukturen löst sich immer weiter auf. Stattdessen wird Arbeit multimobil: Home Office, Remote Work und Vertrauensarbeit heißen die Modelle der Zukunft, welche die Grenzen zwischen Arbeit und Freizeit verwischen. 


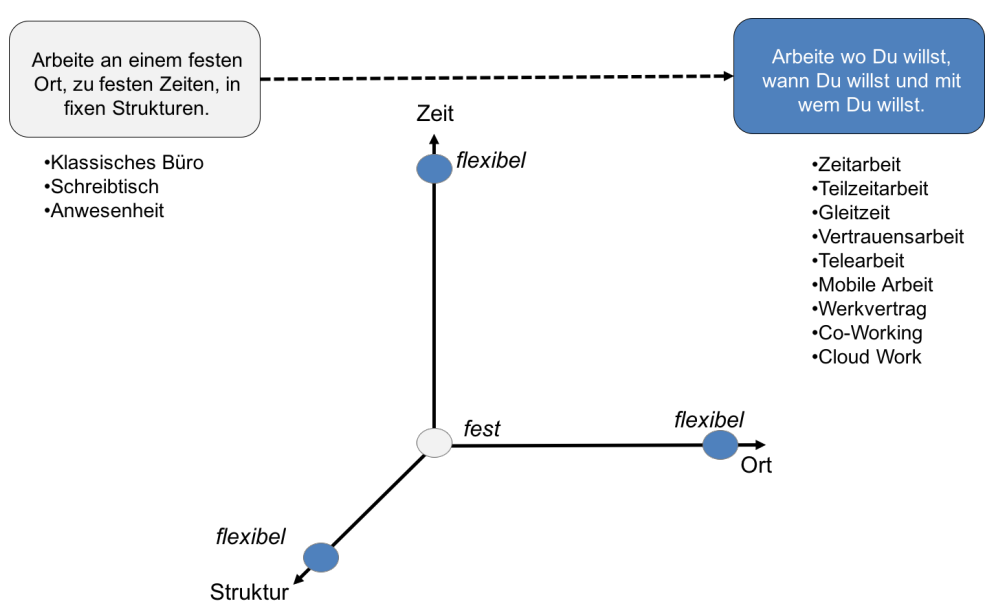

Die Idee der New Work (Eigene Darstellung nach der Idee von

Petry 2016)

Insgesamt zeigt die Grafik von Petry die Vision der New Work. Es bedeutet also: Arbeite wann, wo und mit wem du willst. Klar ist: Die Dimension „Wo“ deutet auf mobile Arbeit und „Wann“ auf eine Lockerung der Kernzeiten hin. Aktuell steht jedoch die Dimension „Mit wem du willst“ noch im offenen Dialog und kann unter anderen durch die Idee des Hashtags Futurework interpretiert werden. Schauen Sie auch in meinen Artikel zu New Work für eine detaillierte Erklärung der Idee.

\section{FUTUREWORK - ARBEIT 4.0}

Insgesamt zeigt sich bei der Untersuchung des Hashtags eine starke Fokussierung auf Raum- und Bürokonzepte zur Förderung von Agilität im Unternehmen. Klar, es wird ebenfalls von einem Kulturwandel gesprochen und dieser auch sehr ausführlich behandelt, aber es werden überwiegend Bürokonzepte evaluiert. So fasst der folgende Satz 
das Thema sehr gut zusammen: „Das Büro wandelt sich zum Ort der Kommunikation und Vernetzung. Dazu werden situationsorientierte Räumlichkeiten benötigt, die sowohl den kreativen Austausch oder die Projektarbeit fördern als auch Rückzugsmöglichkeiten zum konzentrierten und vertraulichen Arbeiten bieten und Routinetätigkeiten optimal unterstützen“ (Quelle: Detecon). In der folgenden Abbildung finden Sie eine Ausgestaltung der Idee mit verschiedenen Büroräumen. Diese sollen ermöglichen, in verschiedenen Teams und verschiedenen Orten zu arbeiten. Schauen Sie auch in meinen Artikel zum Thema Futurework.

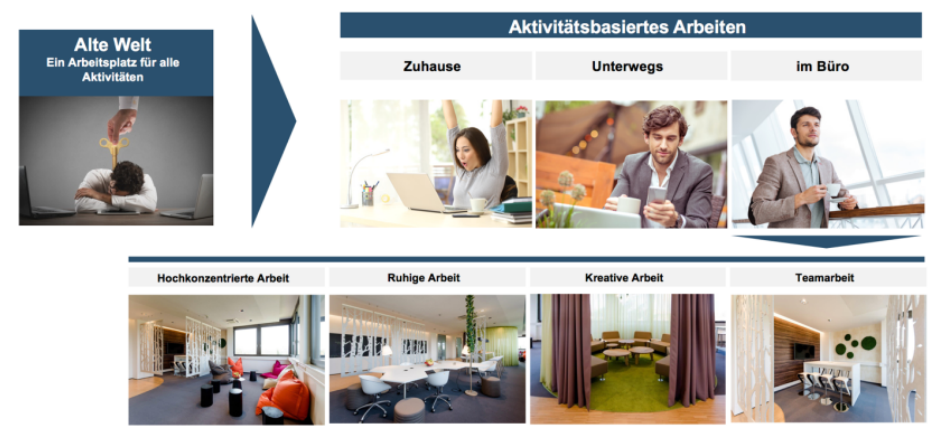

Ausgestaltung des Konzepts der Futurework (eigene Darstellung nach einer Idee der Detecon)

\section{FAZIT: WAS IST ARBEIT 4.0? - DEFINITION}

So wurde in diesem Text die Frage "Was ist Arbeit 4.0?" behandelt. Es zeigt sich, dass der Begriff Hintergrund für eine Ansammlung von Konzepten ist. Arbeit im Kontext der dieser Arbeit 4.0 wird technologisch, agiler, schneller und kurzzyklischer. Enabler für die Arbeit 4.0 ist der digitale Wandel des Arbeitsplatzes, welcher jedoch oft noch am Anfang steht. Ausgestaltet werden kann die Arbeit 4.0 mit 


\section{ARBEIT 4.0}

der Vision der New Work (arbeite wann, wo und mit wem du willst) sowie mit neuen Bürokonzepten.

\section{QUELLEN}

Brandes, U., Gemmer, P., Koschek, H., \& Schültken, L. (2014). Management Y. München: Campus Verlag.

Petry, T. (2016). Digital Leadership: Erfolgreiches Führen in Zeiten der Digital Economy. München: Haufe Verlag.

Detecon:

http://www.detecon.com/sites/default/files/1_DMR_blue_Transforma tion_Neue_Arbeitswelten_D_02_2015_0.pdf

http://www.manager-magazin.de/unternehmen/karriere/digitalisierungder-arbeitswelt-wie-funktioniert-arbeiten-4-0-a-1082272.html

http://blog.wiwo.de/look-at-it/2016/06/16/digitalisierung-am-arbeitsplatzhome-office-meist-besser-ausgestattet-als-buero/

https://newworkblog.de/new-work/ 


\section{DIGITALISIERUNG AM ARBEITS- PLATZ - CHANCEN UND RISIKEN}

\section{Originalartikel: https://agile-unternehmen.de/digitalisierung-am- arbeitsplatz/}

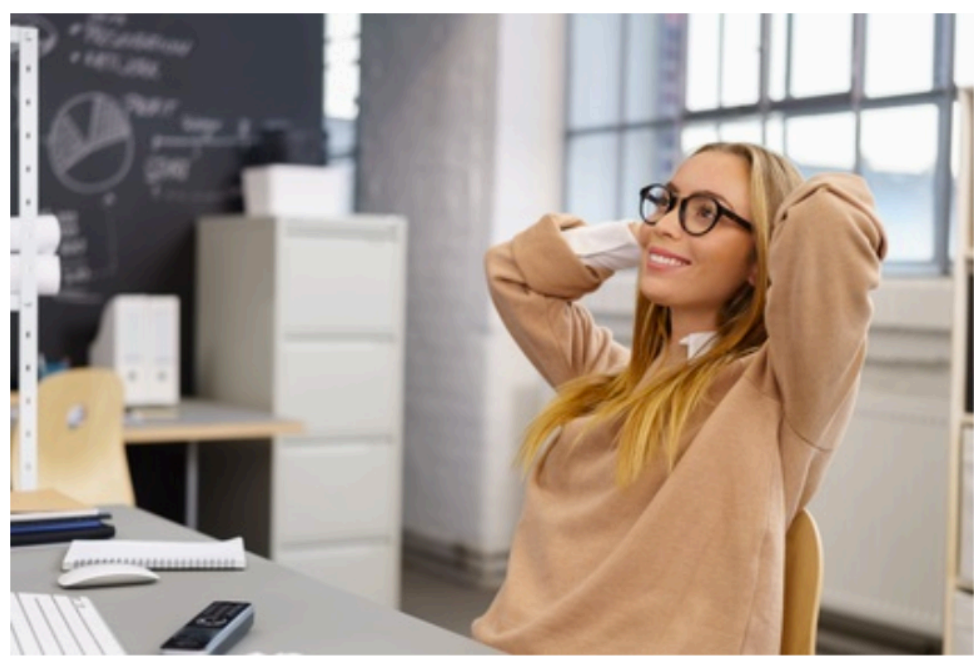

Die Digitalisierung wird das Arbeitsleben in den kommenden Jahren erheblich verändern - in so gut wie allen Bereichen. Wer sich nicht vorbereitet, wird ein Problem bekommen, so ein Artikel der Welt. Der digitale Wandel verändert also zunehmend unser Berufsleben. Ohne Computer und Internet steht die Arbeit heute in vielen Betrieben still. Handwerker fertigen mit digitaler Hilfe Möbel oder Autoteile, Architekten erstellen in Windeseile Baupläne, wo sie vorher aufwendige Zeichnungen anfertigen mussten. Mitarbeiter sind über die sozialen Netzwerke miteinander verbunden, digitale Programme erleichtern Arbeitsabläufe und Absprachen untereinander. Arbeitnehmer können 


\section{ARBEIT 4.0}

sich auf wesentliche Aufgaben konzentrieren und sind beispielsweise nicht mehr mit Verwaltungsaufgaben überlastet (Quelle: Bildungsexperten).

\section{DIGITALISIERUNG AM ARBEITSPLATZ: HOME-OFFICE MEIST BESSER AUSGESTATTET ALS BÜRO}
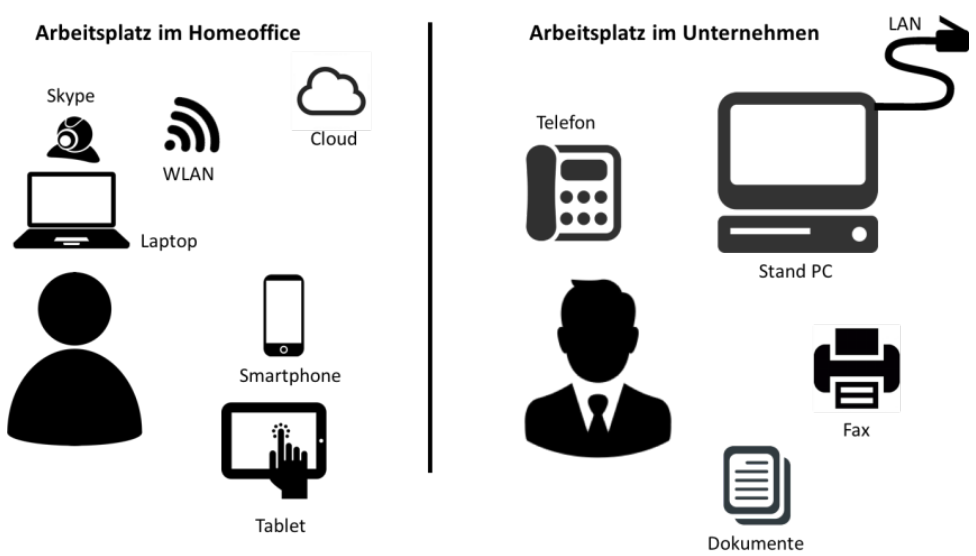

Vergleich Home-Office vs. Arbeitsplatz im Unternehmen (eigene Darstellung, abgeleitet aus der Studie)

Die Studie der WiWo zur Digitalisierung am Arbeitsplatz zeigt zusammenfassend: „Die Digitalisierung ist daheim bereits weiter fortgeschritten als am Arbeitsplatz im Büro das zeigt eine Studie unter deutschen Büroangestellten." So sagt die Studie weiterhin: Der Weg zum wahrhaft digitalen Büro ist noch weit. Denn derzeit ist ein Großteil der deutschen Arbeitsplätze nicht so ausgestattet, dass Wissensarbeiter wirklich digital arbeiten können. Viele administrative Tätigkeiten, die digital deutlich schneller zu bewältigen sind, werden nach wie vor analog ausgeführt. Das 


\section{ARBEIT 4.0}

ist das zentrale Ergebnis einer Studie des HostingDienstleisters Host Europe, die auf einer Umfrage von YouGov unter 1000 Angestellten mit Büroarbeitsplätzen basiert.

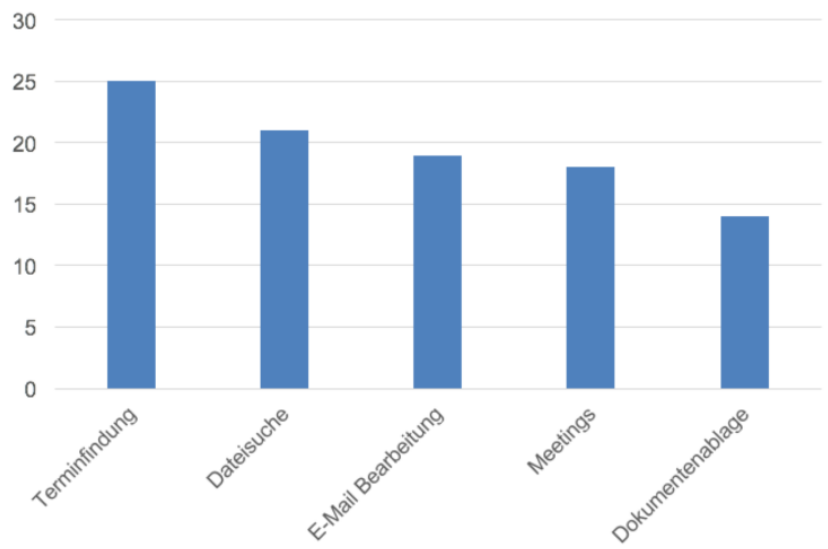

Top 5 der nervigsten Bürotätigkeiten laut der Studie (eigene Darstellung laut der Studie)

\section{STATUS QUO ZUM ZUR DIGITALISIERUNG AM AR- BEITSPLATZ}

Das Bundesministerium für Arbeit und Soziales veröffentlichte einen Digitalisierungsmonitor. Die Ergebnisse der Studie kurz und knapp:

- $\quad$ Ein Großteil aller Befragten in Betrieben mit mindestens 50 sozialversicherungspflichtig Beschäftigten nutzt im Arbeitsalltag digitale Informations- und Kommunikationstechnologien (IKT). 


\section{ARBEIT 4.0}

- Während nur die Hälfte der gering qualifizierten Beschäftigten beruflich IKT nutzt, sind es unter den Hochqualifizierten fast alle.

- Führungskräfte werden deutlich häufiger von ihrem Arbeitgeber mit mobilen Endgeräten ausgestattet als Beschäftigte ohne Führungsverantwortung.

- Knapp vier Fünftel der Beschäftigten haben in den letzten fünf Jahren eine Veränderung in der technischen Ausstattung ihres Arbeitsplatzes erlebt, auch hier steigt der Anteil mit dem Ausbildungslevel.

- Beschäftigte erfahren durch die technologische Veränderung einerseits körperliche Erleichterung und verringerte Anforderungen an die eigenen Fähigkeiten und Kompetenzen, andererseits aber auch die Notwendigkeit, sich weiterzuentwickeln und Multitasking auszuüben.

- Fast 30 Prozent der Beschäftigten berichten von einer körperlichen Entlastung aufgrund technologischer Neuerungen, 15 Prozent von sinkenden Anforderungen an die eigenen Fähigkeiten und Kompetenzen.

- Beschäftigte, die sinkende Anforderungen wahrnehmen, nehmen seltener an beruflicher Weiterbildung teil.

- Fast 80 Prozent der Beschäftigten sehen aufgrund technologischer Veränderungen die Notwendigkeit, die eigenen Fähigkeiten ständig weiterzuentwickeln. 65 Prozent nehmen eine Verdichtung der Arbeit wahr. 
- Beschäftigte, die eine Notwendigkeit zur Weiterentwicklung wahrnehmen, nehmen häufiger an beruflicher Weiterbildung teil.

- 13 Prozent der Beschäftigten sehen es als wahrscheinlich an, dass ihr Arbeitsplatz zukünftig von einer Maschine übernommen wird.

- Etwa ein Drittel der Beschäftigten erlebt eine größere Entscheidungsfreiheit aufgrund technologischer Entwicklungen.

- Mehr als die Hälfte der Befragten nimmt eine Steigerung der eigenen Produktivität wahr.

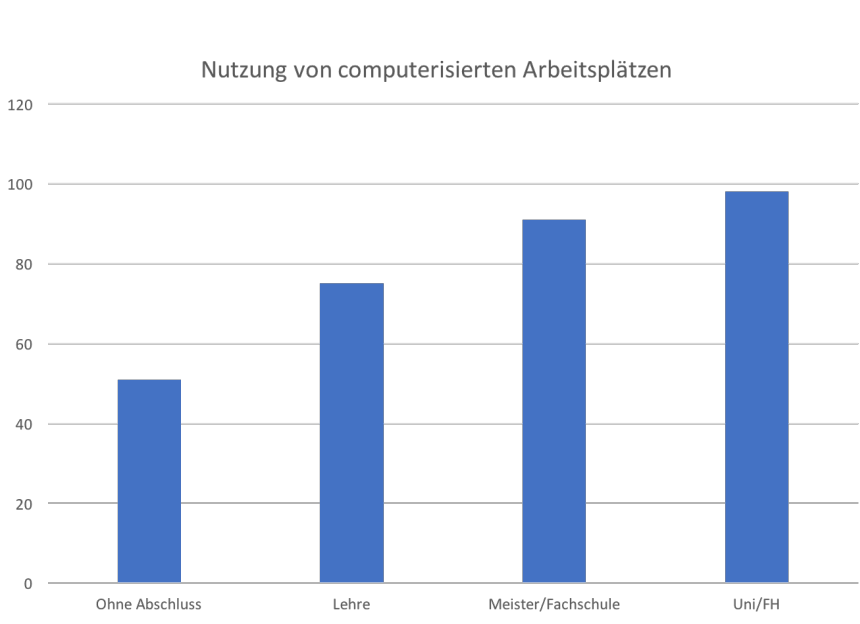

Die Auswirkungen von Technologie am Arbeitsplatz. Wie viel Prozent der Befragten nutzen einen computerisierten Arbeitsplatz nach Bildungsabschluss? Ergebnisse der Studie des BMAS (eigene Darstellung) 


\section{SO VERÄNDERT DIE DIGITALISIERUNG UNSERE AR- BEITSWELT}

In einem Artikel auf Xing werden die Veränderungen durch die Digitalisierung am Arbeitsplatz aufgezeigt. Zuerst nennt der Artikel die steigende räumliche und zeitliche Unabhängigkeit, die daraus resultiert, dass Mitarbeitende über Laptop und andere Mobile Devices ihre Aufgaben nahezu von jedem Ort und immer mehr auch zu flexiblen Uhrzeiten erledigen können. Arbeitnehmende werden somit insgesamt flexibler und können Arbeits- und Privatleben besser miteinander in Einklang bringen.

Weiterhin wurde die Bedeutung von lebenslangem Lernen genannt. Mitarbeitende müssen mehr denn je bereit sein, konstant Neues hinzuzulernen, da die Halbwertszeit von Wissen deutlich abnimmt.

Außerdem kommt eine starke Automatisierung hinzu und erleichtert die Arbeit stark. Der Artikel nennt hierzu: Hierbei sind beispielsweise Ärzte zu nennen, die mittels Augmented-Reality-Brille wichtige Informationen zu ihren Patienten eingeblendet bekommen, während sie operieren, oder Mechaniker, denen die nächsten Schritte für ihre geplante Reparatur virtuell und flexibel angezeigt werden.

Ebenfalls stellt der Artikel eine gesteigerte Komplexität fest und sagt dazu: Informationen werden Mitarbeitenden über eine Vielzahl von Kanälen ununterbrochen zur Verfügung gestellt. Multitasking und die Fähigkeit, sich fokussieren zu können, werden sozusagen Grundvoraussetzung. 
Die Geschwindigkeit im Unternehmen nimmt insgesamt zu - es kommt zu einer stetigen Beschleunigung, auf die sich Mitarbeitende agil und flexibel einstellen müssen.

Zu guter Letzt stellt der Artikel von Xing fest, dass in der digitalisierten Arbeitswelt mehr als bisher in Teamstrukturen gearbeitet wird. Dabei gewinnen vor allem interdisziplinäre Teams an Bedeutung, die sich über verschiedene Unternehmensbereiche oder auch Unternehmensgrenzen hinweg erstrecken, um die besten Lösungen für komplexe Probleme zu erzielen.

Signifikante Veränderung der IT-Ausstattung am Arbeitsplatz

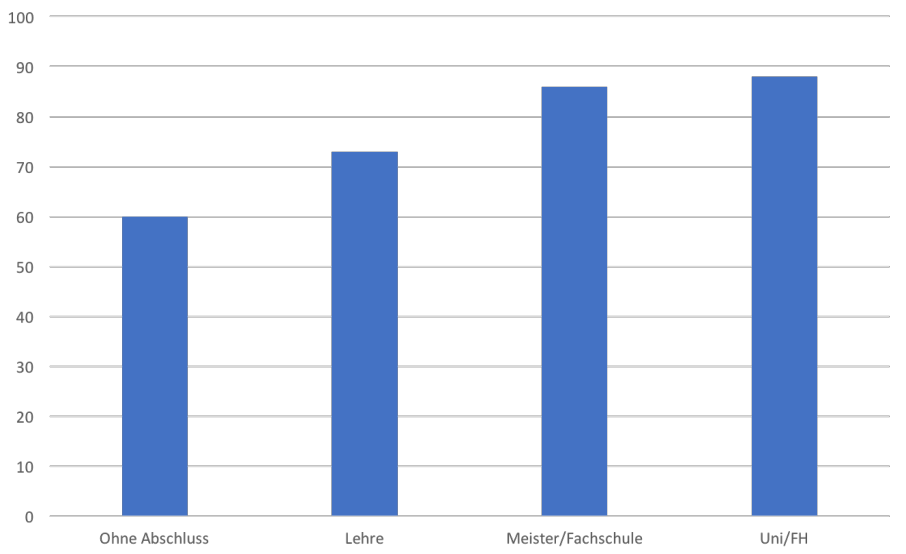

Anzahl der Nennungen in Prozent der Befragten nach Bildungsabschluss, ob sich die IT-Ausstattung am Arbeitsplatz signifikant verändert hat. Ergebnisse der Studie des BMAS (eigene Darstellung) 


\section{CHANCEN DER DIGITALISIERUNG AM ARBEITSPLATZ}

Die Probanden der Studie des BMAS sagen zu den Chancen der Digitalisierung: Die technologischen Neuerungen haben mir mehr Entscheidungsfreiheit zur Gestaltung meiner Arbeit gegeben. Aktuell bestätigen $32 \%$ der Befragten diesem Satz. Ebenfalls zeigt die Grafik unten, dass mittel qualifizierte Frauen die Steigerung der eigenen Arbeitsleistung aufgrund der technologischen Neuerungen etwas häufiger höher empfinden als Männer. Zwischen den Beschäftigten mit unterschiedlichem Ausbildungslevel sowie zwischen den Berufsgruppen sind nur geringe Unterschiede zu beobachten.

Erhöhung der Arbeitsleistung durch technologische Neuerungen

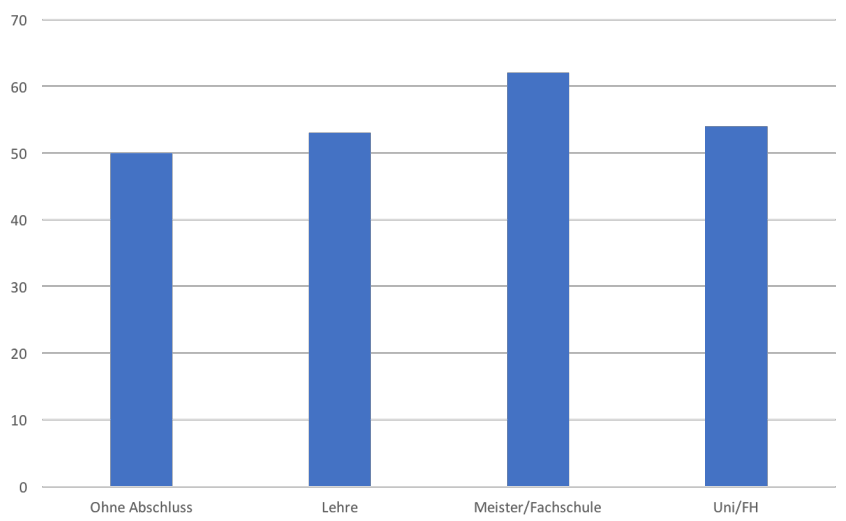

Anzahl der Nennungen nach Bildungsabschluss, ob die bereits vorhandene Digitalisierung die Arbeitsplatzleistung erhöht hat.

Daten anhand der Studie des BMAS (eigene Darstellung) 


\section{RISIKEN DER DIGITALISIERUNG AM ARBEITSPLATZ}

So wie es Chancen gibt, existieren auch Risiken. Der Artikel von Bildungsexperten zeigt diese auf. Erstes Risiko ist eine Abwertung menschlicher Arbeit. Intelligente Software macht menschliche Arbeit in einigen Bereichen überflüssig oder wertet sie finanziell ab. Berufe wie der des Verkäufers, Texters, Sprachlehrers oder Bankangestellten verlieren an gesellschaftlicher und sozialer Bedeutung. So vermitteln Internetplattformen Dienstleister oder Handwerker mittlerweile zu Niedrigpreisen.

Ein weiteres Risiko ist die stressbedingte Erkrankung durch ständige Erreichbarkeit. Arbeitnehmer, die ständig erreichbar sind und immer neue Aufgaben übernehmen müssen, leiden nicht selten unter einer steigenden Arbeitsbelastung. Die Folge sind zunehmende stressbedingte Erkrankungen wie Burn-Out oder Depressionen. Viele Menschen können durch den steigenden Druck nicht mehr von der Arbeit abschalten und denken auch in ihrer Freizeit nur noch an den Job, so die Bildungsexperten. 


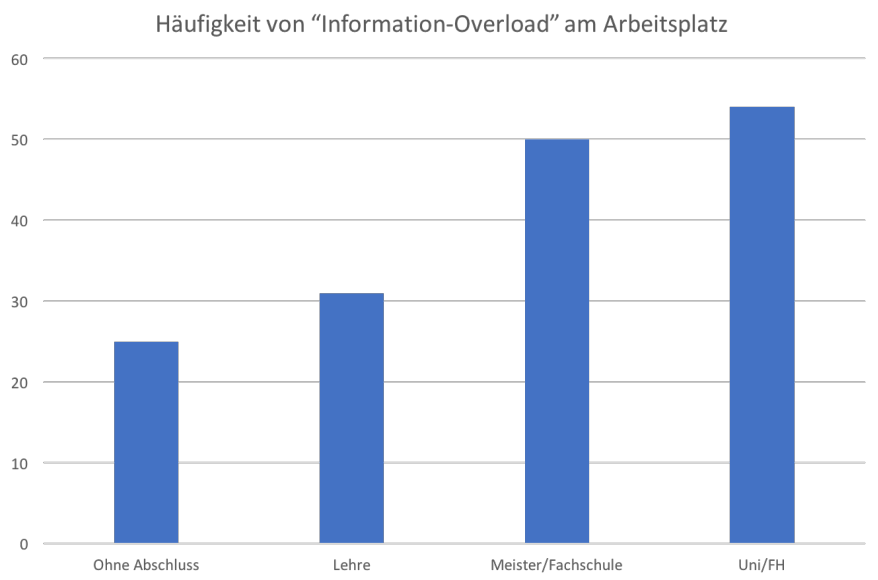

Anzahl der Nennungen nach Bildungsabschluss, ob es eine zu hohe Menge an Informationen am Arbeitsplatz gibt. Daten anhand der Studie des BMAS (eigene Darstellung)

Weiterhin ist Datenschutz ein hohes Risiko. So sagt der Blog von Bildungsexperten: Nicht erst seit den Enthüllungen von Edward Snowden spielt das Thema Datenschutz auch in der digitalisierten Arbeitswelt eine große Rolle. Arbeitnehmer müssen sich darauf verlassen können, dass ihre Daten sicher sind.

Letztes Risiko ist der "gläserne Mitarbeiter“. Hier sagt der Blogartikel der Bildungsexperten: Wer in den sozialen Netzwerken, sei es auf Xing, Twitter oder Facebook, Informationen über sich preisgibt, macht sich unter Umständen zum gläsernen Arbeitnehmer. Im schlimmsten Fall kann er sogar seinem beruflichen Erfolg schaden. Nicht selten nutzen Firmen mittlerweile gezielt die persönlichen Profile ihrer Mitarbeiter, um sich über diese zu informieren und Erkundigungen einzuholen. 


\section{FAZIT: DIE DIGITALISIERUNG AM ARBEITSPLATZ KOMMT!}

In Zukunft wird sich also der Einsatz von digitalen Gadgets am Arbeitsplatz vermehren und die Arbeit des Menschen wird sich durch Automatisierung und intelligenten Maschinen verändern. Menschen werden vermutlich zunehmend Bereiche übernehmen, in denen hohe Kreativität und Denken gefordert ist. Im letzten Teil des Artikels sehen wir, dass die Digitalisierung am Arbeitsplatz auch Risiken birgt. So müssen Mitarbeiter lernen mit Digitalisierung umzugehen und „einfach mal abzuschalten“. Es gilt also eine Digitalkompetenz bei Mitarbeitern und Führungskräften aufzubauen, damit die Digitalisierung den Menschen unterstützten kann, anstatt zur Qual zu werden.

\section{QUELLEN}

https://www.welt.de/wirtschaft/article153754398/Wie-Sie-die-

Digitalisierung-am-Arbeitsplatz-meistern.html

http://www.bildungsxperten.net/job-karriere/arbeit-im-digitalen-wandelchancen-und-risiken/

http://blog.wiwo.de/look-at-it/2016/06/16/digitalisierung-am-arbeitsplatzhome-office-meist-besser-ausgestattet-als-buero/

http://www.bmas.de/SharedDocs/Downloads/DE/PDF-Publikationen/a875monitor-digitalisierung-am-arbeitsplatz.pdf?_blob=publicationFile\&v=2

https://spielraum.xing.com/2016/03/neue-studie-so-veraendert-diedigitalisierung-unsere-arbeitswelt/ 


\section{DIALOG MIT DR. ANDREAS ZEUCH ÜBER DIE „NEUE ARBEIT“}

Originalartikel: https://agile-unternehmen.de/andreas-zeuch-neuearbeit/

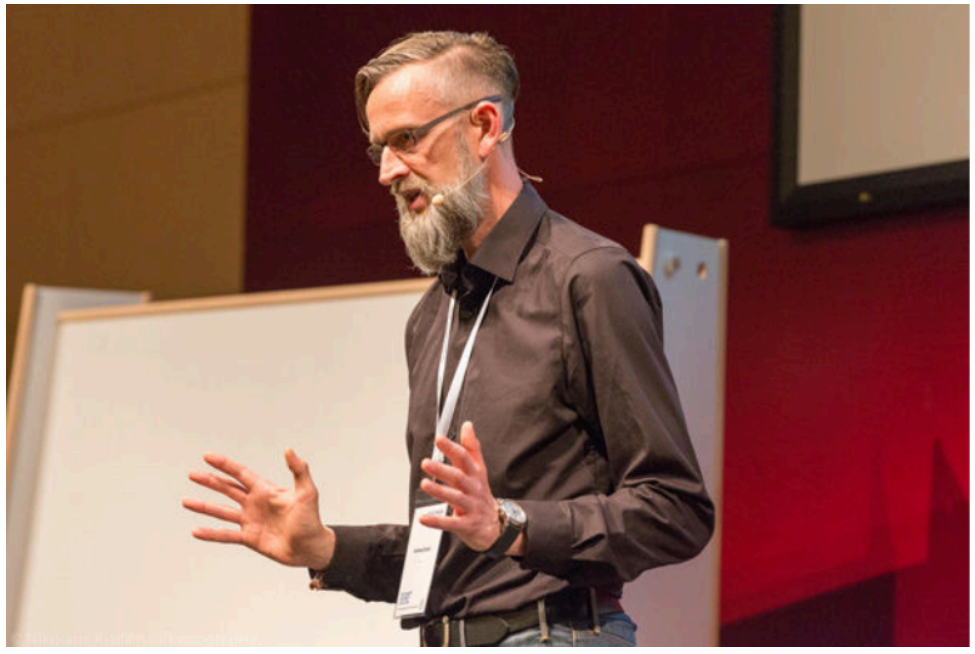

Dr. Andreas Zeuch (*1968) begleitet als Berater, Trainer und Redner Unternehmen auf dem Weg zu mehr Mitbestimmung und Unternehmensdemokratie. 2015 veröffentlichte er sein fünftes Buch „Alle Macht für niemand. Aufbruch der Unternehmensdemokraten“. Er bloggt hierzu immer wieder neue Inhalte auf seiner Homepage zum Thema Unternehmensdemokratie. In diesem Zuge habe ich mich mit inm über vier Themen unterhalten, die in meiner aktuellen Forschung eine große Rolle spielen. 


\section{ANDREAS ZEUCH ÜBER:}

\section{... DIE AKTUELLE SITUATION IN DEUTSCHEN UNTER- NEHMEN}

Zeuch bemängelt aktuell, dass die Mehrheit von Unternehmen in Deutschland oft noch zu "verhaftet“ in alten Modellen von Führung, Kultur und Arbeit sind. Außerdem nimmt er oft Zögerlichkeit im Ausprobieren von neuen Modellen wahr. Als Grund nennt er, dass viele dieser Modelle aus Sicht der Geschäftsführer und Vorstände noch nicht erprobt sind und keine Langzeitstudien vorliegen. Aus diesem Grund hat er in seinem Buch Alle Macht für Niemand erste Beispiele dargelegt, dass alternative Modelle funktionieren können und Unternehmen, die sich demokratisch organisieren, wirtschaftlich sehr erfolgreich sein können. Es ist zwar keine Garantie, aber definitiv eine Alternative zu klassischer Organisation. Schließlich scheitern auch top-down geführte Unternehmen - sogar öfter als demokratische geführte, einfach weil die meisten Firmen herkömmliche Führungsmodelle aufweisen.

\section{... DEN HYPE UM AGILE UNTERNEHMEN}

Oft hört man den Satz "Nur agile Unternehmen sind zukunftsfähig“. Hier widerspricht Zeuch stark und sieht eine Verallgemeinerung fehl am Platz. Als Beispiel nennt er u. a. „stabile Märkte“ wie den örtlichen Nahverkehr. Ein stabiles Umfeld und wenig bis gar keine Konkurrenz verlangen weniger Agilität. In hoch kreativen Bereichen werden agile Unternehmen dennoch einen deutlichen Wettbewerbsvorteil haben. Zeuch fügt jedoch auch hinzu, dass selbst Konzerne, die sich in der einer Krise befinden, oft noch 
jahrelang weiter Geschäft generieren und aktuelle Trends ignorieren können. Klar ist jedoch: „Es kann nicht ewig so weitergehen."

\section{... DIE DIGITALE TRANSFORMATION}

Insgesamt ist die digitale Transformation für Zeuch ein „Buzzword und Hype“. Jedoch sieht er diesen Hype als wichtig an, bemängelt aber den Umgang mit inm. Auf vielen Konferenzen und Think Tanks bemerkt er, dass viele Manager diese Transformation nur von rein technischer Seite betrachten. Deswegen fordert Zeuch eine Konzentration auf die "soziotechnische“ und kulturelle Ebene. Die neue Generation wächst mit digitaler Technologie auf und lernt somit eine neue Art der Kommunikation und des Umgangs mit Technologie. Dies wird zu tiefgreifenden sozialen und kulturellen Änderungen in der Gesellschaft führen. Es liegt an uns, diese nun zu verstehen und darauf zu reagieren, so Zeuch.

\section{... DIE „NEUE ARBEIT“}

Es gibt viele Begriffe für die „Neue Arbeit“, wie Arbeit 4.0, New Work, Futurework usw., so Zeuch. Jedoch sind nicht alle Inhalte neu. Es trifft sogar das Gegenteil zu. Doch oft waren die Konzepte und Methoden zur Zeit ihrer ersten Veröffentlichung noch nicht ausgereift oder noch nicht umsetzbar.

Die Idee hinter der neuen Arbeit ist laut Zeuch einfach: „Jeder, der will, darf mitgestalten, keiner muss, und alle tragen die Konsequenzen." Das ist jedoch nicht so einfach zu pauschalisieren. Es scheint also, über meine subjektiven 


\section{ARBEIT 4.0}

Eindrücke hinaus, eine Entwicklung in Richtung "Neue Arbeit“ zu geben. Der Wunsch nach mehr Partizipation, Mit- und Selbstbestimmung ist dabei keineswegs den angeblich so selbstbestimmungsfreudigen jüngeren Generationen zuzuschreiben, sondern zeigt sich generationenübergreifend, so Zeuch und verweist auf die Studie "Der Ruf nach Freiheit: Innovationsförderliche Arbeitswelten aus Sicht der Arbeitenden" von Hays, der ZukunftsAllianz Arbeit \& Gesellschaft e. V. (ZAAG) und der Gesellschaft für Wissensmanagement e. V. (GWWM). Seine Eindrücke werden gestützt von einer Studie von IBM, die besagt, dass für die Generation $Y$ finanzielle Sicherheit und Arbeitsplatzsicherheit die maßgeblichen Faktoren bei der Wahl des Arbeitgebers sind.

Zeuch nimmt wahr, dass ein Beruf zunehmend mehr als nur "die Ausübung einer Arbeit“ ist. Die Wahlfreiheit im Beruf war nie größer, so Zeuch. Jeder kann sich heute seinen Beruf selbst aussuchen. Sogar psychologische Barrieren wie „Mein Vater war Arzt, und ich werde nun auch Arzt" werden zunehmend abgebaut und jeder ist prinzipiell in der Lage, jeden Beruf zu erlernen - abgesehen von den üblichen Bildungshürden.

\section{FAZIT}

Insgesamt zeigt der der Dialog, dass sich gewisse hoch kreative Bereiche in einem Umbruch befinden. Jedoch sind Agilität und "Neue Arbeit“ keine Garantie für Erfolg, aber eine ernst zu nehmende Alternative. Im Laufe der Forschung wird also weiterhin der sinnvolle Einsatz von agilen Methoden in Unternehmen untersucht. Lesen Sie auch meinen Artikel zur digitalen Arbeit oder den Blog 


\section{ARBEIT 4.0}

von Andreas Zeuch! Ich möchte auch Dr. Andreas Zeuch für den tollen Dialog danken!

\section{QUELLEN}

https://www.935.ibm.com/services/us/gbs/thoughtleadership/millenial workplace 


\section{Kapitel 3 \\ AGILE ORGANISATION}

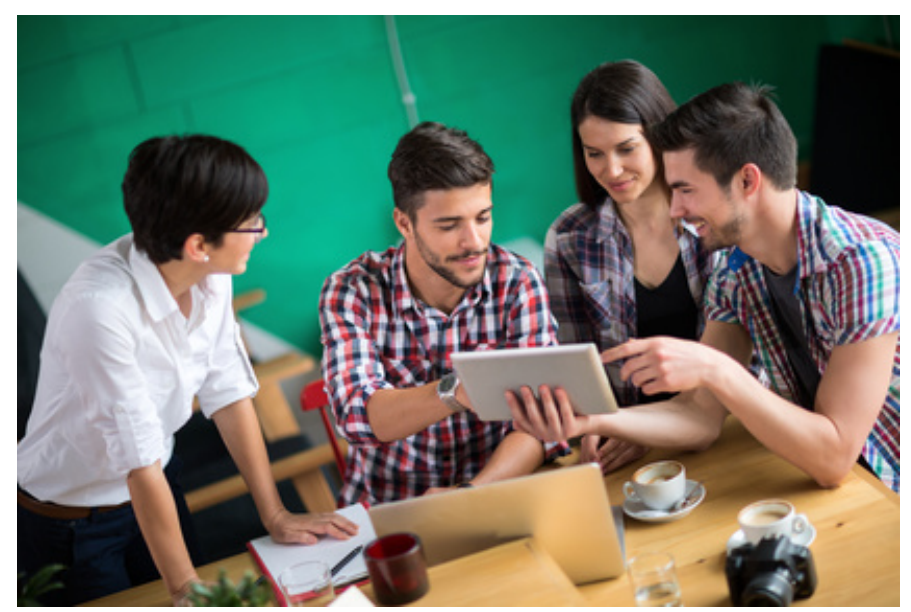

Die digitale Transformation verändert. Sie verändert auch Unternehmen. Diese Veränderungen machen es nötig, dass sich Unternehmen agil den neuen Gegebenheiten anpassen müssen, um nicht von der Konkurrenz abgehängt zu werden. Die Umsetzbarkeit in der Vision des digitalen Wandels in klassischen Unternehmensorganisationsformen ist umstritten, weswegen Unternehmen aktuell eine gesteigerte Agilität anstreben.

Aus diesem Grund habe ich einen Artikel ausgesucht, welcher aufzeigt, was Agilität eigentlich bedeutet, um anschließend aufzuzeigen, wie sich ein agiles Management ausgestalten kann. Am Ende gibt Valentin Nowotny in einem Dialog mit mir einige Tipps zur agilen Organisation. 


\section{WAS IST AGILITÄT?}

Originalartikel: https://agile-unternehmen.de/was-ist-agil-definition/

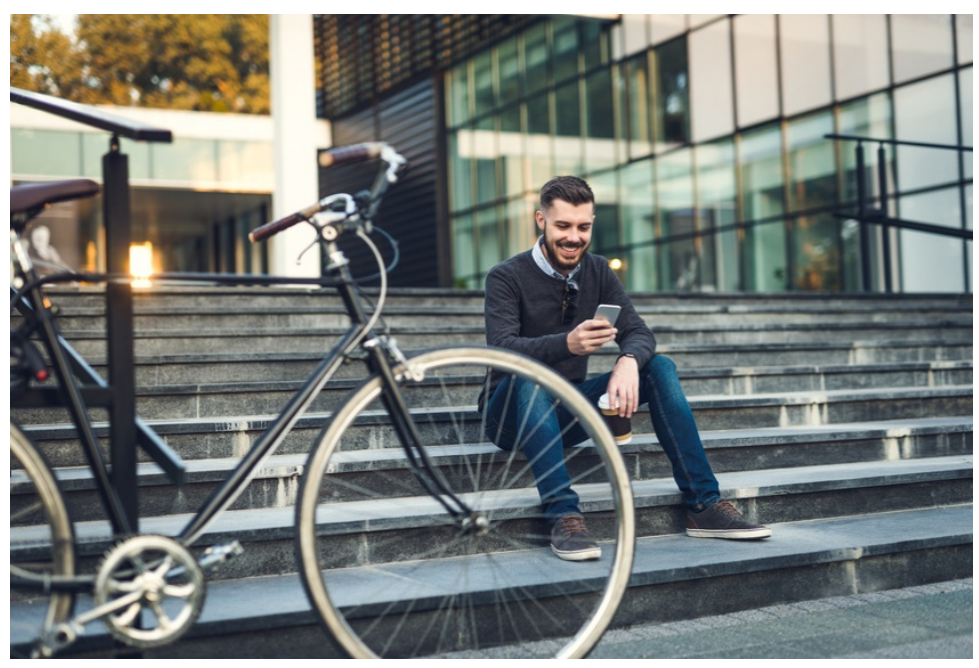

Evolutionäre und agile Unternehmen sind in aller Munde. Doch was ist agil und evolutionär? Was bedeutet agil und evolutionär im Kontext von Unternehmen? Dazu möchte ich versuchen eine erste Definition zu geben.

\section{WAS IST AGIL? AGILE UNTERNEHMEN}

Um Agilität genau zu definieren, habe ich zuerst einmal zahlreiche Bücher angeschaut und Agilität diverse Eigenschaften zugewiesen. Anschließend liefere ich eine 2. Definition aus den typischen Praxisbüchern. 


\section{AGILE ORGANISATION}

\section{DEFINITION AUS DER AKADEMIE}

Agilität ist nicht eindeutig im Kontext von Unternehmen definiert. In nahezu jedem Paper findet man eine neue Definition. Aus diesem Grund habe ich die meist zitierten Veröffentlichungen gesucht und nach Aspekten untersucht, welche diese mit Agilität verbinden. Insgesamt konnten 7 Aspekte gefunden werden, welche in alle 10 Publikationen genannt worden sind.

- Schnelligkeit

- Anpassung

- Flexibilität

- Dynamik

- Vernetzung

- Vertrauen

- Selbstorganisation

Die Eigenschaft Schnelligkeit wird in allen 10 Publikationen in Verbindung mit Agilität genannt. So weisen u. a. Lu und Ramanurthy (2011) Agilität eine schnelle Reaktion auf Veränderung sowie eine schnelle Implementierung von Produkten oder Services mit einer schnellen Änderbarkeit $\mathrm{zu}$. 


\section{AGILE ORGANISATION}

Eine weitere Eigenschaft von Agilität ist Flexibilität. So beschreiben Nissen und Rennenkampff (2013) Agilität mit einer sogenannten Personalflexibilität im Sinne gut ausgebildeter und vielfältig einsetzbarer IT-Mitarbeiter, welche die Reaktion auf unvorhergesehene Änderungen begünstigen. Sambamurthy et al. (2003) sehen außerdem die Umsetzung von änderbaren und flexiblen Lösungen als eine Charakteristik von Agilität.

Agilität ist laut Termer (2016) die Anpassungsfähigkeit an sich verändernde Rahmenbedingungen und neue Marktsituationen. Laut Nissen und Rennenkampff (2013) ist vor allem die kontinuierliche Verbesserung und Flexibilität, welche Agilität bietet, Grundlage für die hohe Anpassungsfähigkeit.

Weiterhin wird Dynamik im Zuge von Agilität genannt. Agilität stellt das Nutzen einer gewissen Form von Beweglichkeit dar (Termer und Nissen 2015). Bewegung im Kontext von Agilität heißt laut Termer und Nissen (2015), dass sich die Stellung eines Menschen in Bezug zu seiner Umgebung im Laufe der Zeit verändert. Agilität bedeutet, diese Beweglichkeit aus einem eigenen Antrieb heraus zu schaffen, in der Regel sogar bevor eine Situation oder ein Geschehen diese Beweglichkeit notwendig bzw. erforderlich machen, so Termer und Nissen (2015).

Agilität benötigt eine hohe Kommunikation in einem Netzwerk aus Individuen (Appelo 2010). Aus diesem Grund misst Appelo (2010) Agilität ebenfalls eine hohe Vernetzung zu, da Eigenschaften wie Flexibilität und Vertrauen 


\section{AGILE ORGANISATION}

auf der Beziehung und der Kommunikation zwischen Individuen aufbauen.

Umgekehrt basiert Vernetzung ebenfalls auf Vertrauen. Dies begründet Termer (2016) daraus, dass flache Hierarchien, autonome Arbeitsgruppen mit kurzen Entscheidungswegen ebenso wie Kreativitätsförderung und eine auf Vertrauen basierende Kultur geschehen müssen.

Selbstorganisation spielt im Kontext von Agilität ebenfalls eine Rolle. Nach Gloger und Rößner (2015) ist Selbstorganisation ein gelenkter und intensiver Lernprozess. Selbstorganisation im Kontext von Agilität ist ebenfalls komplex, menschenorientiert und ein autonom gelenkter und Intensiver Lernprozess.

\section{FAZIT EVOLUTIONÄRE UND AGILE UNTERNEHMEN!}

Es ist klar zu bemerken, dass die Definition noch nicht eindeutig klar ist und wir können noch nicht genau sagen, was agil ist. Es ist ebenfalls unklar, welches Rahmenwerk für Agilität eigentlich nun wirklich zur Definition von agilen Unternehmen passt.

Dennoch ist nun klar, nach welchen Kriterien wir Agilität im Kontext dieses Buchs beurteilen können. Sicherlich gibt es auch noch viel mehr Kriterien zu Agilität, aber vor allem der Einfachheit halber wollen wir es bei diesem 7 belassen. 


\section{AGILE ORGANISATION}

\section{QUELLEN}

Gloger, B., \& Margetich, J. (2014). Das Scrum Prinzip. Stuttgart: Poeschel Verlag.

Laloux, F. (2015). Reinventing Organisations. München: Vahlen Verlag.

Robertson, B. (2015). Holacracy: The New Management System for a Rapidly Changing World. New York: Macmillan USA.

Appelo, J. (2010). Management 3.0: Leading Agile Developers, Developing Agile Leaders. Boston: Addison-Wesley Professional.

Frank, T., \& Nissen, V. (2014). Zum Begriff der Agilität - Betrachtungen und Implikationen aus etymologischer Perspektive.

Gloger, B., \& Rösner, D. (2014). Selbstorganisation braucht Führung. München: Hanser Verlag.

Lu, Y., \& Ramamurthy, K. R. (2011). Understanding the link between information technology capability and organizational agility: an empirical examination. MIS Quarterly, 35(4) 931-954.

Meyer, B. (2014). Agile! The Good, the Hype and the Ugly. Berlin, Heidelberg: Springer.

Nissen, V., \& Rennenkampff, A. (2013). IT-Agilität als strategische Ressource im Wettbewerb. In ClO Handbuch (pp. 1-34). Kissing: Symposion Publishing.

Rennenkampff, A. von (2015). Management von IT-Agilität - Entwicklung eines Kennzahlensystems zur Messung der Agilität von Anwendungslandschaften.

Sambamurthy, V., Bhardadway, A., \& Grover, V. (2002). Shaping Agility through Digital Options: Reconceptualizing the Role of Information Technology in Contemporary Firms. MIS Quarterly, 26(1), 1-14. http://doi.org/10.2307/4132321

Termer, F. (2016). Determinanten der IT-Agilität. Wiesbaden: Springer. http://doi.org/10.1007/978-3-658-14215-5

Zhang, Z., \& Sharifi, H. (2000). A methodology for achieving agility in manufacturing organizations. International Journal of Operations \& Production Management, 20(4), 496-512. http://doi.org/10.1108/01443570010314818 


\section{AGILES MANAGEMENT}

Originalartikel: https://agile-unternehmen.de/agiles-management/

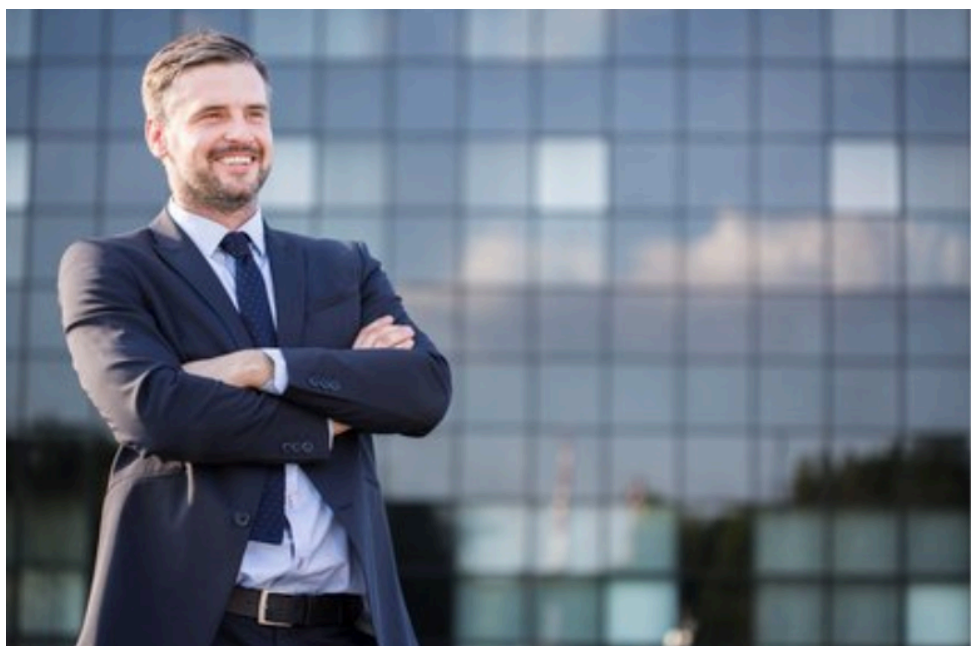

Agile Methoden wie Scrum schaffen Paradigmenwechsel in Unternehmen und revolutionieren somit die eigene Unternehmenskultur. Eigene Glaubenssätze und Verhaltensweisen wie "Das macht man so“ werden infrage gestellt. Gleichzeitig wird die Tür für transparentere Strukturen geöffnet, in denen sich Mitarbeiter stärker einbringen können. Demnach transformieren sich in agilen Unternehmen klassische Positionen zu modernen Rollen.

Bei erfolgreichem Installieren agiler Methoden nimmt das Management eine Schlüsselrolle ein. Damit Führungskräfte, die bisher mit wenig Aufmerksamkeit bedacht wurden, was die Einführung agiler Methoden betrifft, einer solchen Revolution nicht im Wege stehen, brauchen moderne Manager ein deutlich gezeichnetes, neues Bild ihrer eigenen zukünftigen Rolle innerhalb agiler Unternehmen. 


\section{DER MODERNE MANAGER}

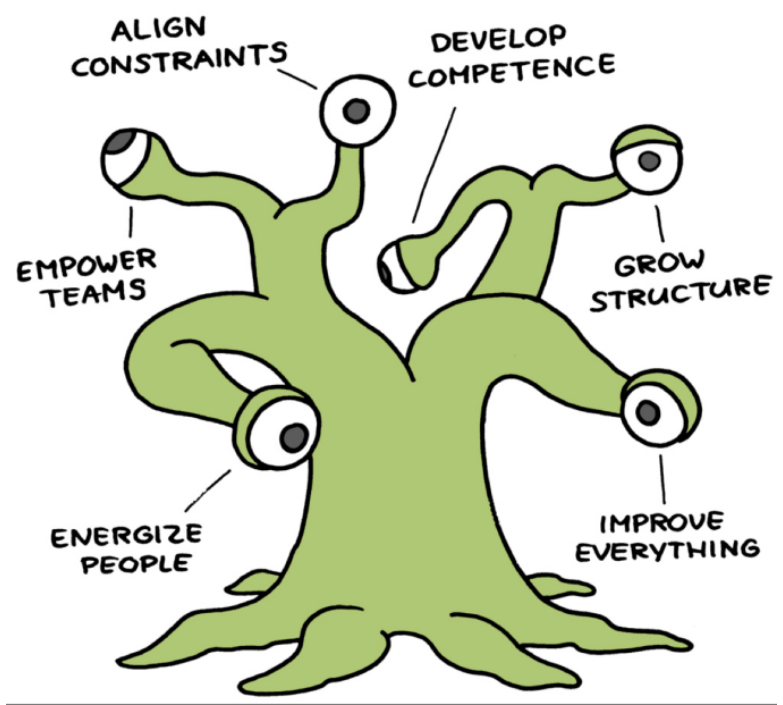

Der moderne Manager: Ein Wesen mit vielen Augen, das bei 360-Grad-Blick das große Ganze im Auge behält und den Fokus auf viele Dinge gleichzeitig richtet. Darstellung: Appelo (2010)

Die Abbildung aus Appelo zeigt den modernen, agilen Manager als ein Wesen mit vielen Augen. Um erfolgreich zu agieren, richtet die Führungskraft den eigenen Fokus auf viele Dinge gleichzeitig. Sie muss nicht nur klare Strukturen schaffen und das agile Team zu jedem Zeitpunkt motivieren, sondern auch die eigenen Kompetenzen erweitern. Durch den Blick auf generelle Neuerungen und Entwicklungen außerhalb der eigenen Firma muss agiles Management auch stets neue Fähigkeiten entwickeln und ist damit immer am Puls der Zeit. Die zahlreichen Augen verschaffen dem Manager dabei einen 360-Grad-Blick, durch den er das große Ganze sieht. 


\section{AGILES MANAGEMENT ENTWICKELT MENSCHEN}

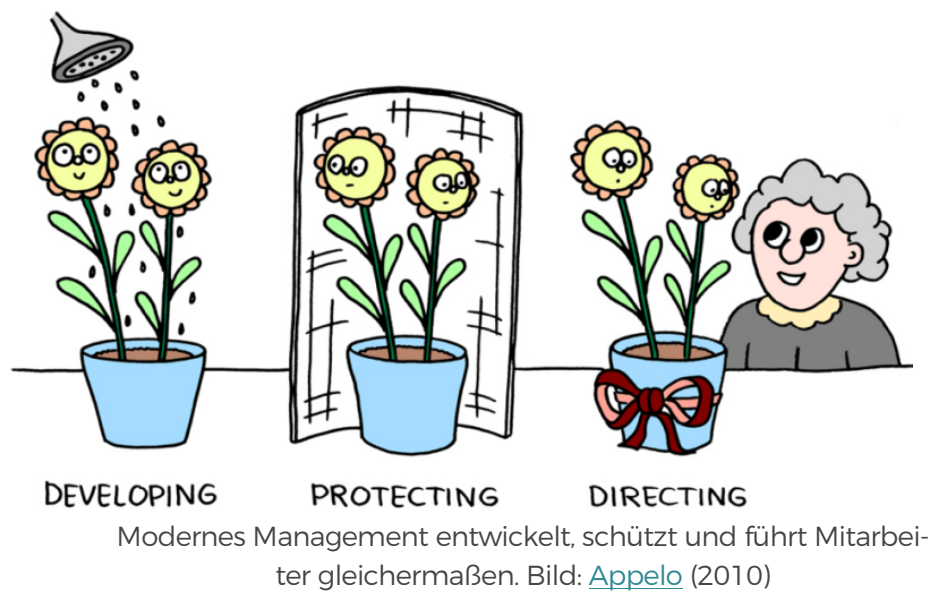

Nach Appelo (2010, S. 155) sind drei der Hauptaufgaben von Managern, Menschen zu entwickeln, die Mitarbeiter zu beschützen und diese bei ihrer persönlichen Entwicklung innerhalb des Unternehmens zu leiten. Wie agiles Human Resource Management es schafft, agile Mitarbeiter zu finden und selbst der neuen Rolle der HR gerecht zu werden, habe ich bereits im Artikel zu agiler HR behandelt. 


\section{KOMPLEXITÄT IM MANAGEMENT}

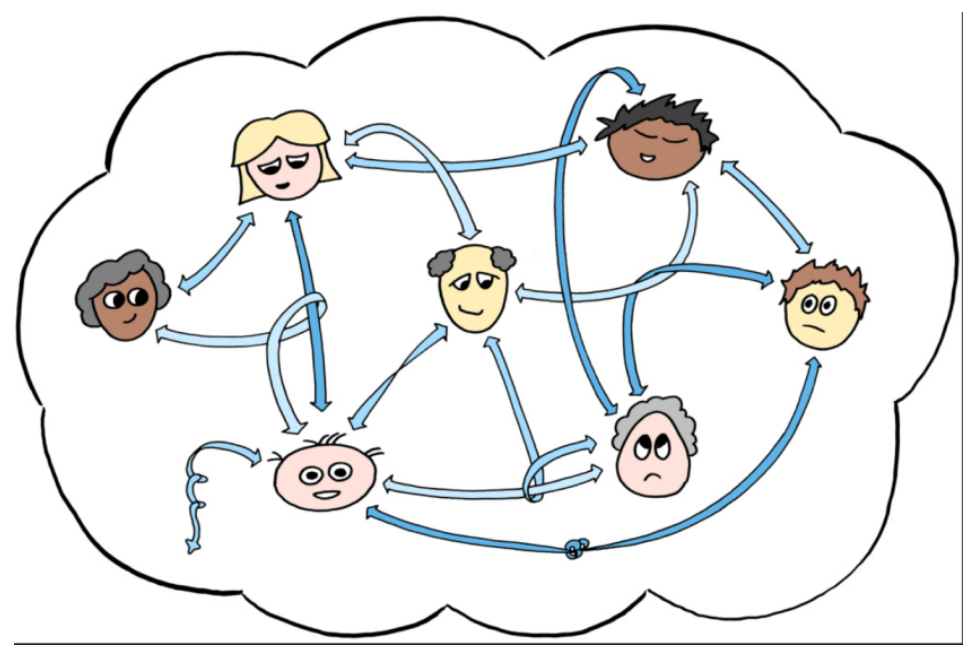

Die komplexe Wirklichkeit führt bei vielen Managern zu einer „Entscheidungslethargie“. Agile Methoden können Abhilfe schaffen. Darstellung: Appelo (2010)

Die Abbildung aus Appelo (2010, S. 52) verdeutlicht die komplexen Systeme, denen sich Führungskräfte ausgesetzt fühlen. Nicht selten führt diese Komplexität zu einer Lethargie hinsichtlich wichtiger Entscheidungsprozesse. Entscheidungen werden, wenn überhaupt, häufig erst zur Deadline getroffen. Moderne, agile Methoden wie Selbstorganisation und Holacracy können die Komplexität aufweichen und die Möglichkeit zur Entscheidungsfindung transparenter machen. 
Agiles Management muss ein ausgewogenes Verhältnis zwischen Entscheidungsfreiheit für Mitarbeiter und klaren Anordnungen schaffen (Appelo 2010)

Modelle wie Holakratie (siehe dazu meinen Artikel zu Holakratie) können allerdings schnell in eine Art Anarchie abdriften, was der Unternehmenskultur sicher nicht gut tut. Auf der anderen Seite will man jedoch weg von alten, festgefahrenen Strukturen und eben jene zu strenge, intransparente Ordnung aufweichen. Die Rolle von agilem Management besteht also darin, das Chaos zu regieren und eine Ordnung innerhalb der Holacracy zu schaffen. Anders gesagt: Agiles Management schafft ein ausgewogenes, perfektes Verhältnis zwischen Anarchie und Diktatur. Lesen Sie auch meinen Artikel zu Komplexität im Management.

\section{AGILES MANAGEMENT GEGEN KOMPLEXITÄT}

Die meisten Organisationen sind nach einer klassischen Hierarchie aufgestellt. Diese ist laut Appelo nicht agil und wird eigentlich auch nicht gelebt. Deswegen fordert Appelo also eine Hierarchie für Organisation und eine informelle Struktur für das Netzwerk im Unternehmen. So ist jeder einzelne Mitarbeiter der Organisation mit den anderen vernetzt. 


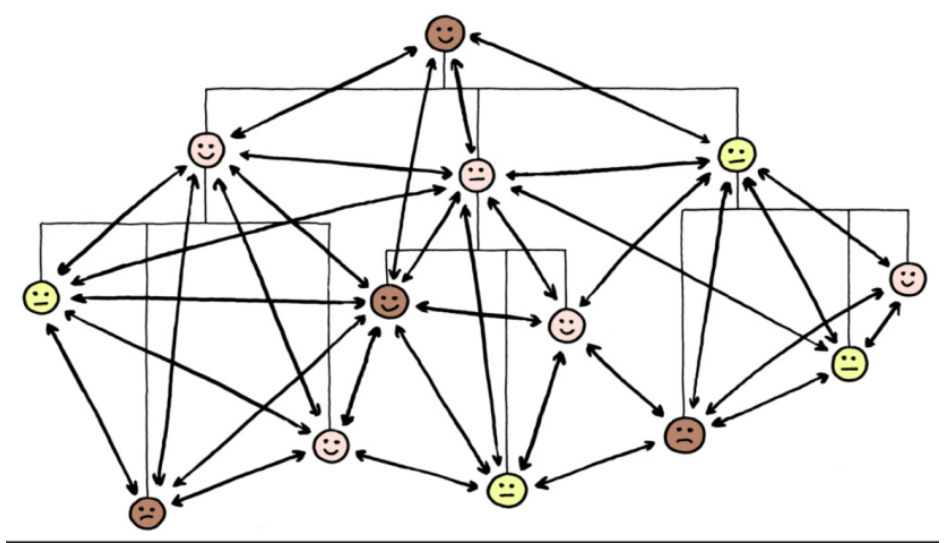

Ein Netzwerk für die Kommunikation und eine Hierarchie für die Führung (Appelo 2010).

Appelo schlägt zur Lösung ebenfalls einen Ansatz vor, welchen er als agil definiert. So ist die Idee, dass Organisationen grundlegend ein klassisches Projektgeschäft etablieren. Diese werden von internen Dienstleistern unterstützt. Dies sind spezialisierte Teams wie z. B. ein GUI-Team. Diese Teams verfügen über ein agiles Mindset, welches darauf beruht einen ausgezeichneten Service für die ProjektTeams zu bieten und diese so gut es geht zu unterstützen. Ein ähnlicher Ansatz wurde ebenfalls im 2. Roundtable zu digitalen Unternehmen konzeptioniert. 


\section{AGILE ORGANISATION}

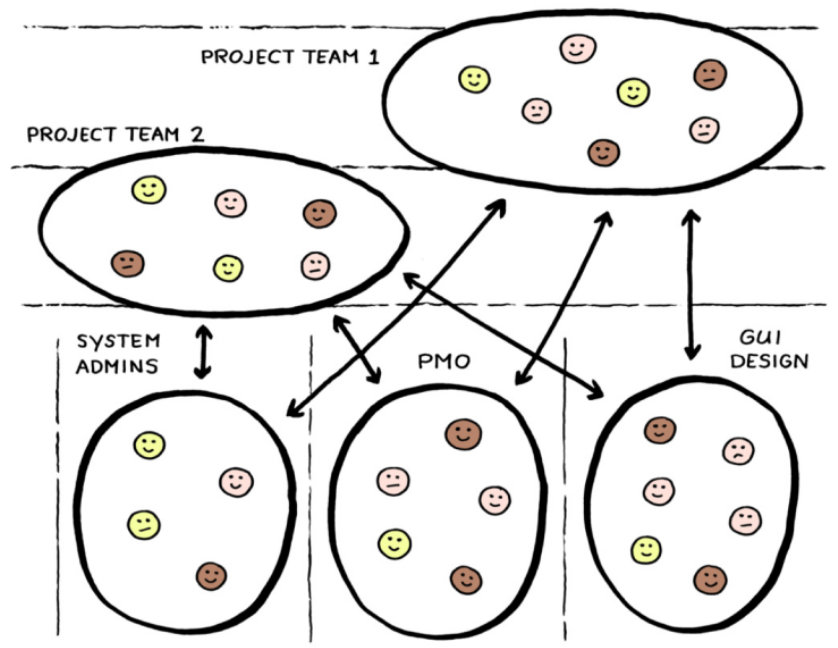

Spezialisierte Teams in der Organisation verstehen sich als „interne Dienstleister“, welche die Organisation unterstützen (Appelo 2010).

\section{STOPLERSTEINE FÜR AGILES MANAGEMENT}

Doch die Umsetzung von agilem Management ist nicht einfach und verläuft nicht immer problemlos. So sagt der Harvard Business Manager: Nicht selten sind Teams mit der Umstellung auf agile Managementsysteme jedoch überfordert. Denn mehr Freiheit bedeutet eben auch, offen zu sagen, wenn etwas schlecht läuft - und Kritik von Gleichgestellten auszuhalten, wenn Ergebnisse hinter den Erwartungen zurückbleiben. Wer unnötige Reibungsverluste vermeiden will, sollte deshalb vor der Transformation klare Regeln für die Zusammenarbeit definieren - auch was Urlaub, Gehälter und Arbeitszeiten angeht.

Das Magazin führt also menschliche Sorgen oder den Verlust von Status an. Oft sind Mitarbeiter in einer gewissen Routine, welche Sicherheit bietet und glauben mit dem 


\section{AGILE ORGANISATION}

agilen Wandel ihre Position zu verschlechtern oder haben generell Angst vor der hohen Unsicherheit einer möglichen Selbstorganisation. Agilität benötigt also andere Fähigkeiten als die Arbeit in einer klassischen Hierarchie. So sagt das Magazin am Ende zusammenfassend: Manager, die ihre Organisation umstellen wollen, sollten daher darauf achten, ob die Mitarbeiter die nötigen Voraussetzungen erfüllen.

\section{ZUSAMMENFASSUNG}

Kein Zweifel - das traditionelle Management steckt in der Krise. In einer immer komplexeren und wandlungsfreudigeren Welt scheinen Managementprinzipien, die im letzten Jahrhundert in vielen Bereichen durchaus erfolgreich waren, immer mehr an ihre Grenzen zu stoßen. Immer offensichtlicher wird, dass ein Führungsverständnis, das auf individuellen Vorgaben und Kontrolle beruht, in der heutigen Zeit nicht mehr funktioniert - in einer Zeit, in der es vorrangig um kreative Wissensarbeit, um komplexe Aufgabenstellungen und um flexibles Reagieren auf Veränderungen im Markt geht.

Zu limitieren ist, dass Appelo seinen Ansatz im Jahr 2010 entwickelt hat und dieser bereits sehr ausgereift ist. Durch neue Erkenntnisse ist es nun möglich auf seinen Erkenntnissen aufzubauen und mit neuen Ansätzen das Modell zu erweitern. Im Verlauf der Forschung wird sich zeigen, wie diese Ansätze Unternehmen helfen können, sich in einer digitalen Welt zukunftsfähig zu wandeln und mithilfe von Agilität und Veränderung auf die massive Geschwindigkeit des technischen Fortschritts zu reagieren. 


\section{AGILE ORGANISATION}

\section{QUELLEN}

Appelo, J. (2010). Management 3.0: Leading Agile Developers, Developing

Agile Leaders. Boston: Addison-Wesley Professional.

https://www.flickr.com/photos/jurgenappelo/sets/72157625328824303/wit h/5201252369/

http://www.harvardbusinessmanager.de/blogs/woran-agiles-managementscheitern-kann-a-1091729.html 


\section{DIALOG MIT VALENTIN NOWOT- NY: DIE AGILE ORGANISATION}

Originalartikel: https://agile-unternehmen.de/valentin-nowotny-agileorganisation/

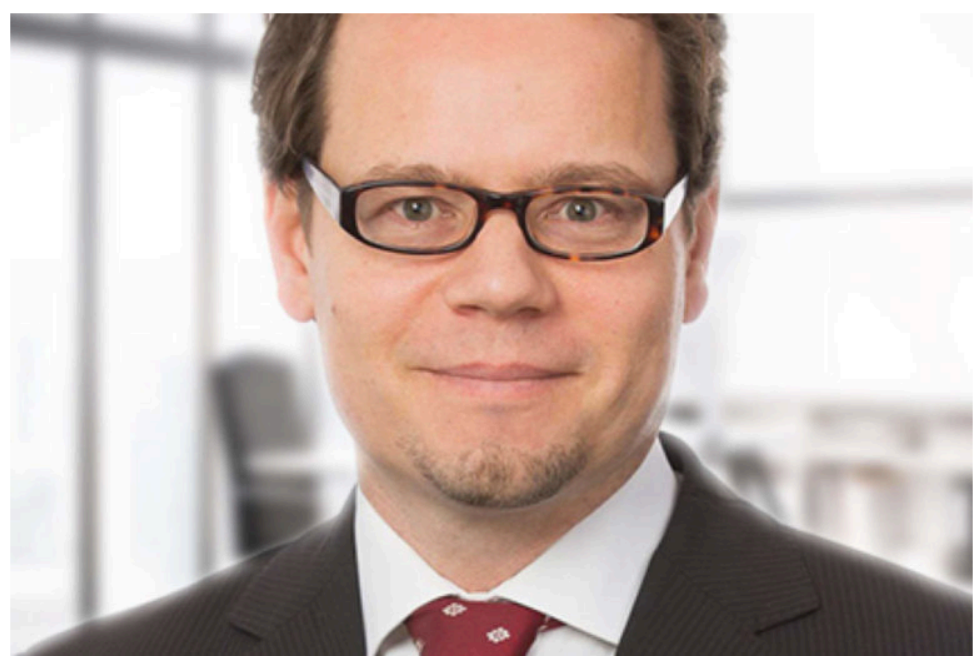

Im Zuge der Forschungsarbeit habe ich zahlreiche Bücher und Quellen zitiert. Nun war es mein Anliegen, auch die Gesichter der wichtigsten Publikationen kennenzulernen und mich mit diesen auszutauschen. So habe ich einen Dialog mit Valentin Nowotny zum Thema Agile Organisation geführt. Valentin Nowotny ist Autor des Buches Agile Unternehmen - nur was sich bewegt, kann sich verbessern. Der Diplom-Psychologe und langjährige Projektmanager arbeitet nun als Berater zum Thema Agile Organisation. Im Zuge des Projekts habe ich mich mit Valentin Nowotny über Thesen meiner Promotion unterhalten und veröffentliche hier die Zusammenfassung des Gesprächs. 


\section{AGILE ORGANISATION}

\section{WAS BEDEUTET AGILITÄT?}

Im ersten Teil haben wir geklärt, was Agilität eigentlich bedeutet und kamen auf 4 wesentliche Punkte: Mindset, Werte, Prinzipien und Praktiken. Laut Nowotny sind Mindset und Werte sowie viele Prinzipien von Agilität bereits definiert. Wo sich jedoch die Literatur noch streitet sind im Bereich der Ausführung, eben der Praktiken. Hier gibt es unendlich viele Tools und Methoden. Es gibt hier kein richtig oder falsch.

\section{UNTERNEHMEN BESCHÄFTIGEN SICH MIT AGILITÄT}

Auf die Frage "Was machen Unternehmen eigentlich gerade?" sagt Nowotny: "Sie wollen agiler werden“. Aktuell befassen sich Unternehmen also mit solchen agilen Praktiken und überlegen: „Wie können wir diese umsetzen, wie fühlt sich das, ist es das richtige für uns?“ Nowotny begleitet hier Teams und sieht als kritischen Erfolgsfaktor aktuell ebenfalls, dass Menschen miteinander reden müssen. Er sagte dazu:

Menschen müssen sich mit anderen Menschen auseinandersetzen. Teamprozesse sind wichtiger denn je.

\section{AGILE UNTERNEHMEN BEWEGEN SICH}

Der Titel von Nowotnys Buch lautet: Nur was sich bewegt, kann sich verbessern. Hier meint Nowotny bewusst auf das Wort „verändern“ verzichtet zu haben. Denn ebenfalls Dinge, welche sich nicht bewegen, verändern sich. Sie werden 


\section{AGILE ORGANISATION}

z. B. alt. Wenn sich etwas jedoch verbessern will, dann muss es sich bewegen.

Doch wohin soll es sich bewegen? Eine agile Organisation ist laut Nowotny fokussiert, schnell und flexibel. Agilität ist nicht radikal, sondern ein konstanter Prozess der Veränderung. Viele Unternehmen sind bereits sehr erfolgreich, jedoch müssen sich auch diese verbessern, um langfristig erfolgreich zu sein.

\section{NUR EINE AGILE ORGANISATION KANN LANGFRISTIG ERFOLGREICH SEIN!}

Kurzfristig kann diese These als falsch erachtet werden. Viele Unternehmen sind momentan erfolgreich und fahren hohe Umsätze ein. Jedoch kann mittel- und vor allem langfristig diese These richtig sein, so Nowotny. Aktuell ist Agilität noch unsicher: Zum Beispiel sind Praktiken wie Selbstorganisation von Teams noch nicht erforscht und agiles Führen wird noch genau definiert. Doch Praktiken von heute müssen nicht zwangsläufig auch morgen erfolgreich sein. Eine agile Organisation wie Apple ist mittlerweile eines der wertvollsten Unternehmen der Welt. Deutsche Unternehmen probieren oft noch zu wenig. Eine Ausnahme ist hier der deutsche Mittelstand („Hidden Champions“). Dieser strebt nach globalen Märkten und Kooperationen mit anderen Mittelständlern und hat damit den DAX-Konzernen einiges voraus. 


\section{AGILE ORGANISATION}

\section{STATE OF THE ART IN UNTERNEHMEN}

Ich habe im letzten Teil des Dialogs mit Valentin Nowotny über den aktuellen Stand in Unternehmen gesprochen. Er selbst meint, dass die Aufgaben in kleinen Unternehmen spannend sind, aber dort oft die Professionalität fehlt. Geht man in größere Unternehmen, dann ist es zwar professionell aber es fehlt die Spannung und das Vertrauen, so Nowotny. Er fordert, dass Arbeit nicht zwingend „professionell“ sein sollte, sondern „sich richtig anfühlen soll“. Nur wenn ein Projekt laut den Guidelines erfüllt worden ist, fühlt es sich nicht automatisch richtig an. Er stellt dort folgenden Satz fest:

Viele arbeiten 8 Stunden und machen Dienst nach Vorschrift und gehen voller Elan nach Hause um dort z. B. Forschung zu betreiben und sich selbst zu verwirklichen.

Laut Nowotny ist Deutschland durch viele kleine und groBe Erfindungen wirtschaftlich bedeutsam geworden. Doch mittlerweile fokussieren sich Menschen zu wenig auf ihre Arbeit als wirkliche schöpferische Herausforderung. Agilität soll diese Lust zurückbringen und wieder mit Leidenschaft und Pioniergeist kreative Prozesse anstoßen. Nowotny stellt aus eigener Erfahrung fest, dass es sehr frustrierend ist, Anträge über 3 Hierarchiestufen genehmigen zu lassen. So verlieren Mitarbeiter Motivation oder arbeiten sogar in einer Parallelwelt. Nowotny meint hiermit: Auf der einen Seite erledigen diese die von der Organisation geforderte Arbeit, um dann „hintenrum“ den Kunden heimlich Nutzen zu stiften. 


\section{AGILE ORGANISATION}

Wir haben in den Unternehmen Mitarbeiter, welche für eine halbe Million Euro Häuser bauen und hier als Bauherr sehr verantwortungsvoll agieren, und sich dann wegen einer Ausgabe über z. B. 50 Euro im Unternehmen mehrfach absichern müssen, warum diese unumgänglich war.

Vielen Dank an Valentin Nowotny für den spannenden Dialog. 


\section{Kapitel 4 \\ DIGITAL LEADERSHIP}

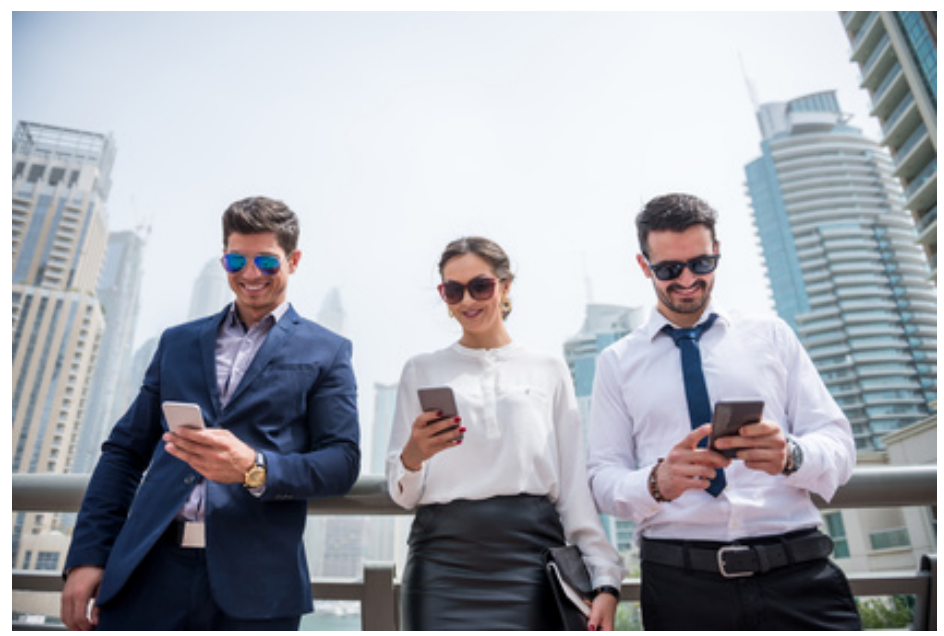

Auf die neue Generation an Führungskräften wird eine Reihe hoher Erwartungen projiziert. Neben einer Vielzahl an Wunsch-Eigenschaften muss ein digitaler Leader immer den passenden Führungsstil für jeden Mitarbeiter aufweisen und den Spagat zwischen Steuerbarkeit und Kontrolle im Unternehmen wahren. Offenheit und Augenhöhe statt Command and Control.

In diesem Kapitel habe ich zuerst einen Artikel ausgewählt, welcher aufzeigt, was Digital Leadership eigentlich ist, um anschließend die Eigenschaften eines Digital Leader genau zu beleuchten. Es zeigt sich, dass viele Parallelen zum agilen Leadership existieren. Der Dialog mit Svenja Hofert zeigt anschließend praktische Tipps auf, wie ein agiles oder digitales Leadership umgesetzt werden kann. 


\section{WAS IST EIGENTLICH DIGITAL LEADERSHIP?}

Originalartikel: https://agile-unternehmen.de/was-ist-digitalleadership/

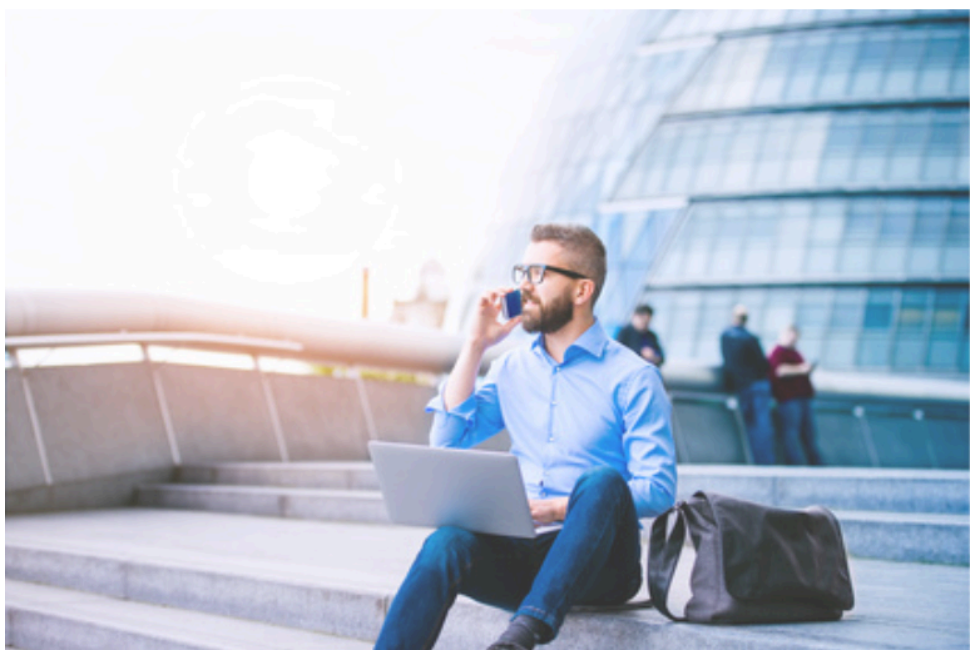

Digital Leadership ist ein wissenschaftlicher Ansatz zur Definition der Aufgaben und Werkzeuge der Führung in Zeiten der Digitalisierung allgemein und in Phasen der Transformation in die Digitalisierung im Speziellen. Diese Definition findet man aktuell bei Wikipedia. Doch was ist nun genau anders an dieser Führung in der digitalen Welt? Bereits das Forschungsprojekt des Crisp stellte Folgendes fest:

Auf die neue Generation an Führungskräften wird eine Reihe hoher Erwartungen projiziert. Neben einer Vielzahl an Wunsch-Eigenschaften pflegt der Digital Leader 


\section{DIGITAL LEADERSHIP}

vor allem einen konstruktiven und kontinuierlichen Austausch mit der eigenen IT-Abteilung, informiert sich aktiv zu IT-Innovationen und denkt disruptiv mit Blick auf neue Prozesse und Geschäftsmodelle.

\section{WAS ZEICHNET EINEN DIGITAL LEADER AUS?}

Führung gibt es schon lange und es gibt zahlreiche Konzepte um agile Führung, digitale Führung und klassische Führung im Sinne des Taylorismus (Command and Control). In meiner Recherche bin ich auf 9 Führungsstile gestoßen, welche $90 \%$ der möglichen Verhaltensweisen einer Führungskraft abdecken sollten. Im Wesentlichen möchte ich als Ansatz des Digital Leadership speziell auf einen davon eingehen, nämlich das partizipierende Führen.
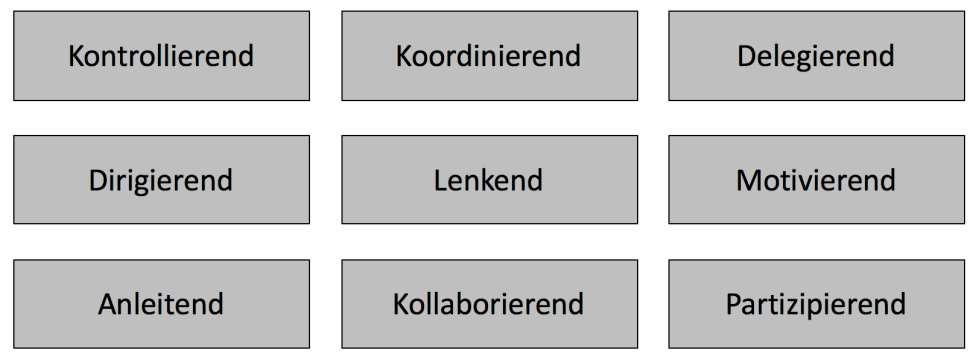

Führungsstile im Team (eigene Darstellung basierend auf dem Wissen von Karrierebibel)

\section{PARTIZIPIERENDES FÜHREN IM TEAM}

Unter den genannten Führungsstilen hat sich also das partizipierende Führung in den aktuellen Newsartikeln als Ansatz zur Ausgestaltung der Vision des Digital Leadership durchgesetzt. So gibt das Magazin Karrierebibel folgende Handlungsempfehlungen zur Umsetzung dieser Führung: 


\section{DIGITAL LEADERSHIP}

- $\quad$ Ein Digital Leader muss ein Unternehmen in die Digitale Transformation führen, das heißt den Wandel aktiv gestalten und dafür auch selbst offen sein.

- Digital Leaders müssen insgesamt mehr Flexibilität zeigen, das heißt auf veränderte Situationen auch entsprechend reagieren können.

- $\quad$ Ein Digital Leader sollte geschulte Medienkompetenz besitzen und in der Lage sein, dieses Wissen seinen Mitarbeitern zu vermitteln.

- Eine digitale Führungskraft braucht ein gutes Gefühl für Teambuilding: Man muss als Chef nicht alles wissen, aber man muss sich die Leute ins Team holen, die das erforderliche Know-how mitbringen.

- Damit einher geht ein Verständnis von Diversity: Im Unternehmen arbeiten verschiedene Generationen, Menschen mit unterschiedlichen Ideen und Hintergründen zusammen, die unterschiedliche Werte verkörpern und unterschiedliche Arbeitsweisen pflegen. Das muss organisiert werden, damit der Wandel nicht in Chaos und Anarchie mündet.

- Digital Leaders benötigen aber auch die nötige Kompetenz und Entscheidungsmacht, die Unternehmensstruktur insgesamt zu verändern und womöglich völlig neue Teams zu bilden.

- Und sie brauchen Budgets, Zeit, Geduld und Fingerspitzengefühl: So ein radikaler Wandel ist kein Sprint. Schon gar keiner, der nur verordnet werden kann. Die Mitarbeiter müssen dazu ermuntert werden, brauchen Vorbilder und ein klares und für sie ebenso lohnendes Ziel. Wer fürchten muss, dass er sich bei der Transformation nur selber abschafft, ist kaum zu motivieren. 


\section{DIGITAL LEADERSHIP}

\section{DER CHIEF DIGITAL OFFICER}

Ein weiterer Ansatz stellt neben digitalem Führen auch die Rolle eines digitalen Leaders dar. Das Beratungshaus Deloitte untersuchte in der Studie mit 102 Unternehmen die Rolle des Chief Digital Officer und was dieser können sollte. Ergebnis war Folgendes: Der CDO ist nicht nur das Bindeglied zwischen allen Führungskräften der Vorstandsebene, sondern auch der direkte Draht zur gesamten Organisation und den Mitarbeitern. Er schafft eine klare Vision für das gesamte Unternehmen, bricht bestehende Silos auf und bewirkt die notwendigen Veränderungen in der Organisation, um die bevorstehende Mission zu meistern. Die digitale Transformation stellt den CDO vor große organisatorische und persönliche Herausforderungen und erfordert daher auch eine herausragende Führungspersönlichkeit, die nicht leicht zu finden ist.
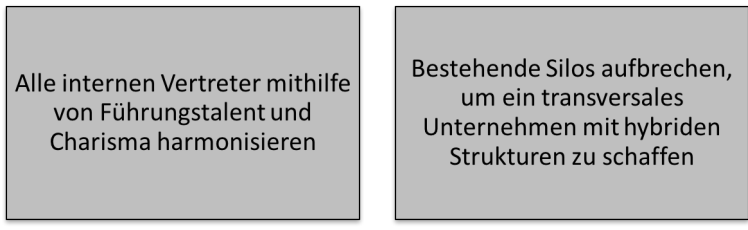

Die bestehende

Unternehmenspolitik verstehen und diese aktiv durch sein Wissen und seine Erfahrungen in verschiedenen Bereichen steuern

Ein fundiertes Wissen in den Bereichen E-Commerce, Marketing, Social Media, Mobile, Big Data und digitale Technologien besitzen

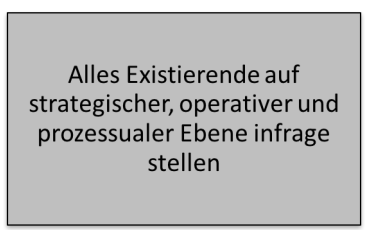

Traditionelle

Wertschöpfungsketten verstehen (Dienstleistungen und/oder Produktion) sowie über relevante operative Erfahrungen verfügen

Die Rolle des Chief Digital Officer (eigene Darstellung basierend auf den Ideen von Deloitte) 


\section{DIGITAL LEADERSHIP}

\section{SPANNUNGSFELD DES DIGITAL LEADERSHIP}

Im Januar 2017 habe ich bereits einen Roundtable zum Thema der digitalen Führung abgehalten. Es zeigt sich, dass es im Wesentlichen 2 Spannungsfelder dieser Leader gibt: zum einen die Frage nach der Balance zwischen Autonomie und Vertrauen und auf der anderen Seite zwischen der digitalen und der analogen Welt.

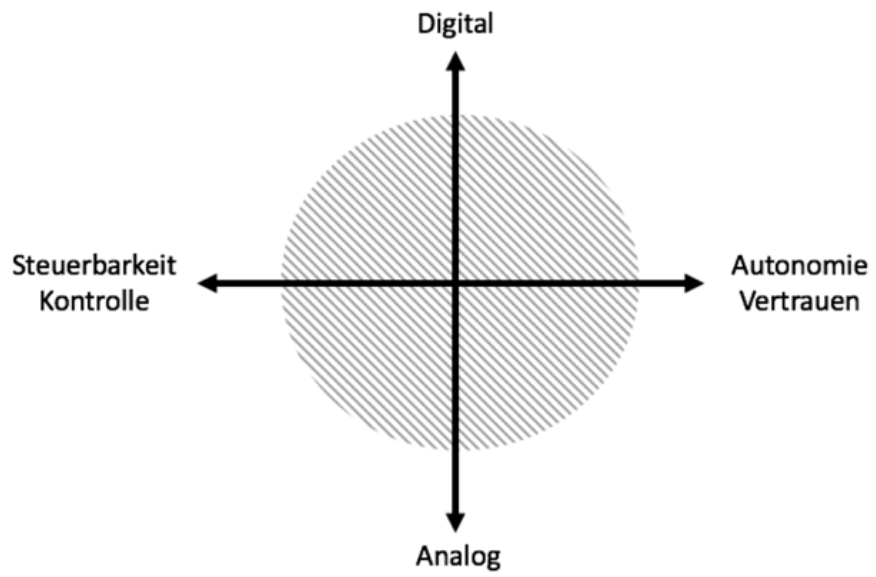

Spannungsfeld der digitalen Leader (Lindner \& Greff 2017)

Spannungsfeld 1 ist also die Frage nach Autonomie und Freiraum für die Mitarbeiter. Der Konsequenz aus dem Roundtable war recht eindeutig.

Einerseits wollen die Führungskräfte Mitarbeitern mehr Freiraum geben und andererseits ist ein Mindestmaß an Kontrolle nötig, um Anarchie zu vermeiden! 


\section{DIGITAL LEADERSHIP}

Auf der anderen Seite rechnen aktuell Führungskräfte mit einem Clash der Generationen. So trifft bereits die digital zurückhaltende Generation $X$ auf die digital geprägte Generation Y. Hinzu wird noch kommen, dass beide auf die vollkommen digitale Generation $Z$ treffen werden. Nicht jede dieser Generationen kann mit den gleichen Methoden geführt werden und es bedarf also eines Spagat für den Digital Leader. Lesen Sie mehr dazu im Artikel zum 4. Roundtable.

\section{FAZIT: ALTER WEIN IN NEUEN SCHLÄUCHEN}

Das Zitat geht auf die Wendung in der Bibel nach Matthäus 9,17 zurück. Die bereits bekannten Führungsstile bleiben also weiterhin erhalten und Führungskräfte sollten diese im Sinne einer digitalen Welt also nur gezielt zur richtigen Zeit einsetzen. So kann eine Führungskraft nicht mehr ausschließlich einen Stil bevorzugen, sondern muss sich aus der breiten Palette bedienen, um den Herausforderungen der digitalen Welt zu begegnen.

\section{BEST-PRACTICE: SITUATIV FÜHREN}

Eine Führungskraft soll also immer wieder gezielt Führungsmethoden individuell passend für jeden Mitarbeiter einsetzen. Eine tolle Best-Practice dazu habe ich im Magazin Karrierebibel gefunden. Das Magazin sagt dazu: Nach einem situativen Führungskonzept müsste auf die Bedürfnisse der einzelnen Mitarbeiter eingegangen werden. Der neue Mitarbeiter braucht beispielsweise noch eine genauere Einweisung, da er sich noch nicht auskennt und unsicher bei seinen Aufgaben ist. Ein Kollege, der die nötige Erfahrung bereits hat, würde auf der anderen Seite mehr 


\section{DIGITAL LEADERSHIP}

Eigenverantwortung bekommen. Diese Flexibilität ist der große Vorteil eines situativen Führungsstils. In der folgenden Grafik finden sich die Führungsstile und welche Reifegrade diese voraussetzen. So ist beim Dirigieren noch eine hohe Kontrolle durch detaillierte Vorgaben vorhanden, wohingegen beim Überzeugen schon die Stimme des Mitarbeiters einbezogen wird. Beim Teilhaben wird der Mitarbeiter ermutigt eigenverantwortlich zu handeln und beim Delegieren wird die Verantwortung komplett an diesen abgegeben.

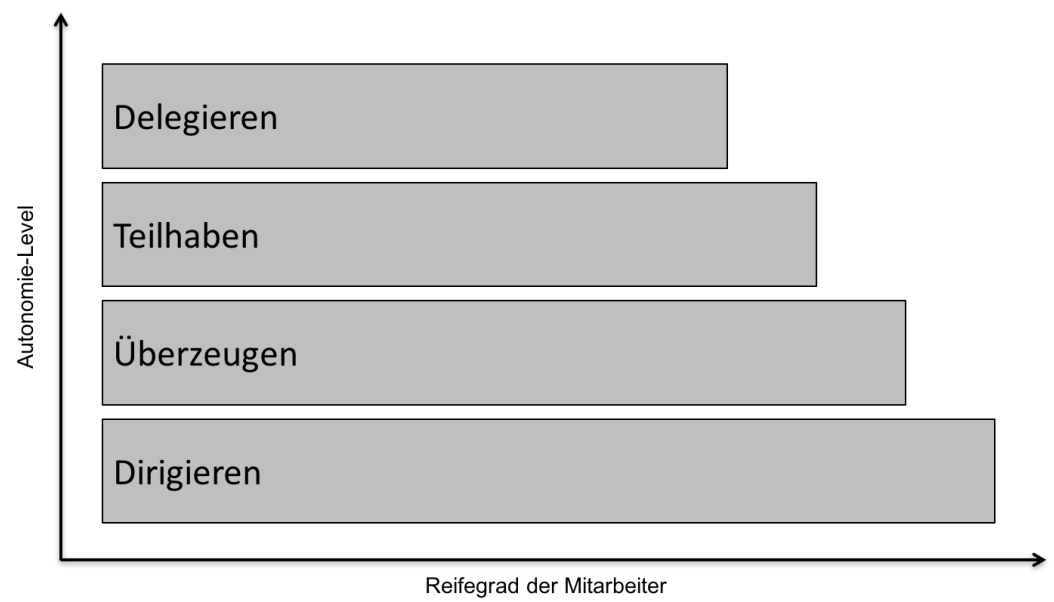

Führungsstile sowie der Grad an Autonomie (eigene Darstellung basierend auf dem Wissen von Karrierebibel) 


\section{DIGITAL LEADERSHIP}

\section{QUELLEN}

https://de.wikipedia.org/wiki/Digital_Leadership

https://www.crisp-research.com/publication/digital-leader/

http://karrierebibel.de/digital-leadership/

https://www2.deloitte.com/de/de/pages/technology/articles/survival-

through-digital-leadership.html

http://karrierebibel.de/situativer-fuehrungsstil/ 


\section{DIGITALE FÜHRUNG IM TECHNO- LOGISCHEN WANDEL}

Originalartikel: https://agile-unternehmen.de/digitale-fuehrung/

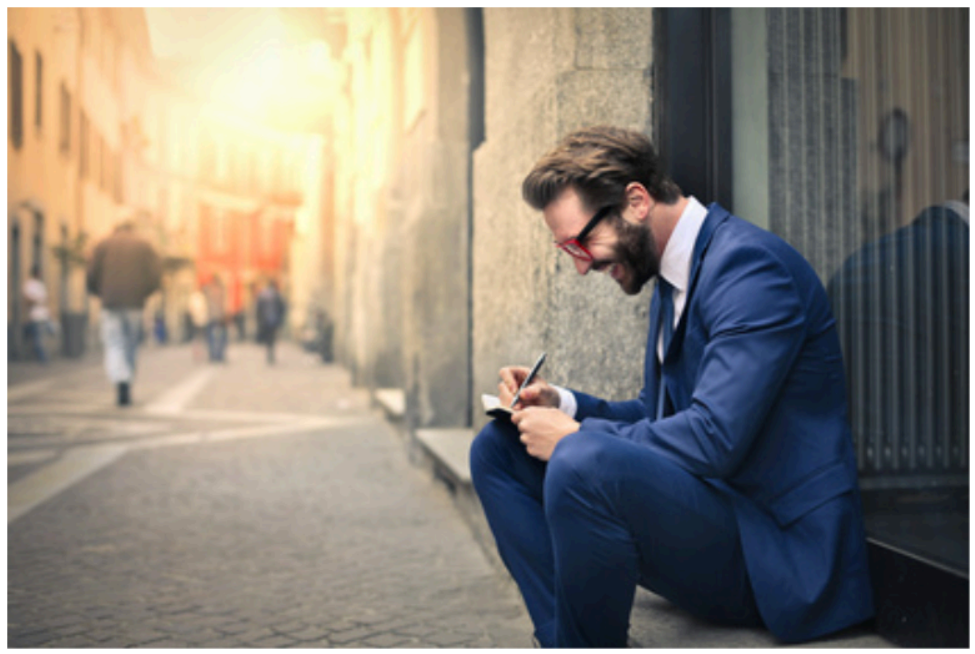

Welchen Einfluss hat die digitale (R)evolution auf Führung? Thema eines Artikels der Hannover Messe war der Einfluss genau dieses Wandels. So heißt im Einladungstext: Die Erfahrung zeigt, dass Unternehmen zugleich innovativ sowie traditionsbewusst sind. In vielen Bereichen sind diese Unternehmen keine technologischen First Mover, sondern beäugen mit einiger Zurückhaltung manche Trends und Entwicklungen. Im Kontext der Digitalisierung gilt es, eine Balance zwischen gebotener Innovation und gewünschter Tradition zu finden. Betroffen sind Kunden, Unternehmen, Führung und die eigene Rolle. In diesem Artikel soll sich auf das Thema „Digitale Führung - Wie 


\section{DIGITAL LEADERSHIP}

verändert diese unsere Zusammenarbeit?“ konzentriert werden.

\section{HERAUSFORDERUNG: FÜHRUNG IM DIGITALEN ZEIT- ALTER}

Flache Hierarchie, neue Arbeitsweisen, Umgang mit Meinungsführern in Organisationen ... Die neuen Herausforderungen für Führungskräfte sind vielfältig. In der Abbildung sind diese etwas detaillierter dargestellt. Es zeigt sich, dass Menschen in Netzwerken zusammenarbeiten und Führungskräfte diese kontrollieren müssen. Kontrolle in Form von Wissensaufbau: Individuelles Wissen soll zu kollektiven Wissen werden. Die bekannte Hierarchie wird zu unbürokratischen und flachen Hierarchien. Dadurch übernehmen Experten und Meinungsführer den Einfluss in der Organisation auf sich und neue Arbeitsweisen wie Scrum und Co. werden ausprobiert. Die für mich größte Herausforderung ist allerdings die Schnelligkeit der digitalen Transformation. Ich merke es bereits beim Bloggen: jeden Tag kommen neue Themen, Wissen und Fragen ans Tageslicht, welche bearbeitet werden können. Die Geschwindigkeit ist enorm und erste Stimmen werden laut: Es ändert sich schneller, als wir uns ändern können. 


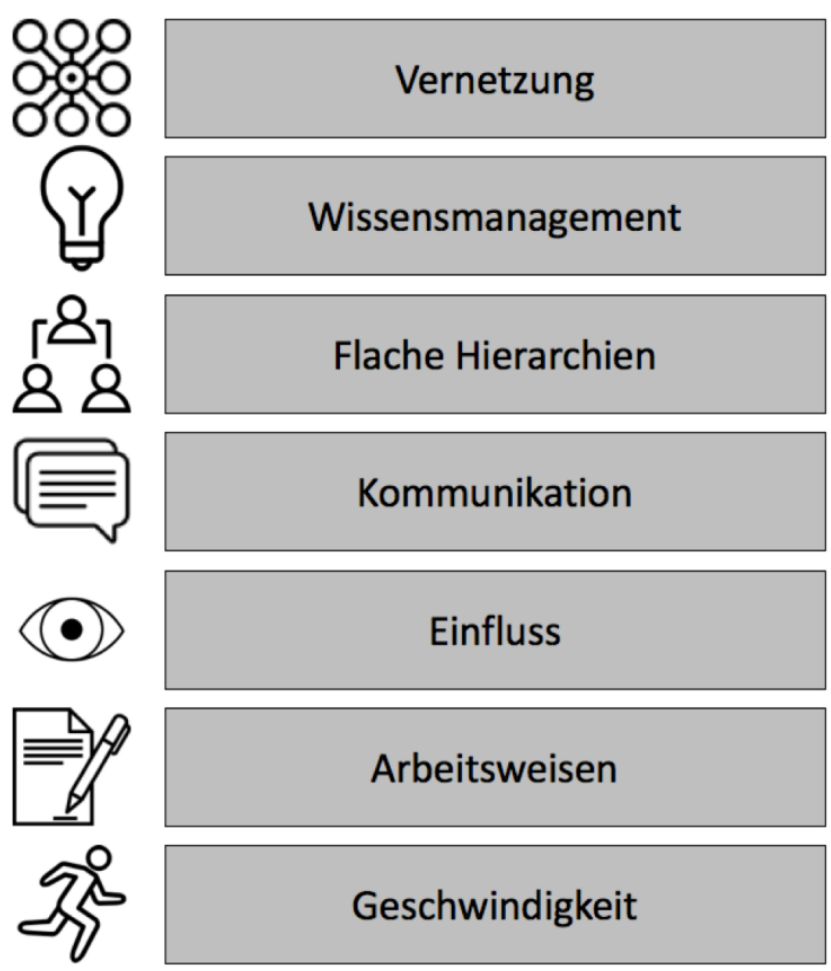

Digitale Unternehmen stehen im Kern vor 7 Herausforderungen, welche sich durch die digitale Transformation ergeben (eigene Darstellung nach den Ideen von Capgemini).

\section{DIGITALES BEWUSSTSEIN FÖRDERN}

„Entscheider müssen die Digitalisierung zu einem Teil ihrer DNA machen. Unternehmen und ihre Mitarbeiter sollten auf allen Ebenen digital denken und handeln", so das Consultinghaus Accenture. Führungskräfte sollen also in erster Linie digitales Bewusstsein fördern und Vorreiter im Unternehmen identifizieren, gemeinsam mit diesen Vorreitern dann durch ständiges Ausprobieren „auf Tempo kommen“ und dauerhaftes Engagement sichern. Erfolgsfaktor ist ein 


\section{DIGITAL LEADERSHIP}

neues Führungsverhalten, welches kollektiv Verantwortung verteilt. Doch wie sieht das genau aus und wie verändern sich die Aufgaben von digitalen Führungskräften?

\section{AUFGABEN FÜR DIE DIGITALE FÜHRUNG}

Die zentrale Frage in diesem Szenario ist: Was macht eine digitale Führung? Welche Aufgaben hat diese inne? Laut der Studie von Capgemini sind dies 6 zentrale Punkte, welche in der Abbildung dargestellt sind. Zusammenfassend lässt sich sagen, dass die Führungskraft vorantreibt, anerkennt, Orientierung gibt, Zusammenarbeit fördert, aber dennoch Entscheidungen trifft. Besonders gefällt mir an dieser Studie, dass alles im organisatorischen Rahmen stattfindet. Dies ist ein immer wieder vergessenes Szenario, denn diese Rahmen sind uns allen gegeben. Wir müssen versuchen in diesen Rahmen das Beste aus uns herauszuholen. Was jedoch aktuell in der Forschung hinterfragt wird: Sind diese Rahmen nicht vielleicht etwas zu eng? Dies wird jedoch in meinen Artikeln zu Digital Leadership und den Interviews zu Agilität thematisiert. 


\section{DIGITAL LEADERSHIP}

\section{Digitale Führung}

Veränderungen vorantreiben

Leistung anerkennen

\section{Zusammenarbeit fördern}

Mitarbeiter entwickeln

Entscheidungen treffen

\section{Orientierung geben}

Digitale Führung hat laut einer Studie von Capgemini 6 Aufgaben, welche sich in den Rahmenbedingungen der Organisation bewegen (eigene Darstellung).

\section{TIPPS: FÜHRUNG IM DIGITALEN ZEITALTER}

Führung im digitalen Zeitalter führt also zu neuen Herausforderungen und einer neuen Beschreibung von Führung. Diese Punkte wurden bereits im Artikel in den ersten Ansätzen geklärt. Doch eine Frage bleibt vor allem für den Praktiker offen: Und wie geht das jetzt? Hierzu habe ich verschiedene Artikel gelesen und daraus 7 Tipps abgeleitet. Natürlich sind diese nicht vollständig, aber sicherlich ein erster Schritt. 


\section{DIGITAL LEADERSHIP}

1. Rolle als Digital Leader bewusst machen: Im ersten Schritt sollte sich jeder Manager mit der digitalen Transformation vertraut machen. Was bedeutet dieser Trend eigentlich und wie kann er in meinen Unternehmen Anwendung finden? Es muss klar sein, dass es keine eindeutige Definition dieses Trends gibt. Die Schlüsselfrage ist: Was bedeutet er im Kontext meines Unternehmens? Hilfe gibt es im Artikel Was ist die digitale Transformation?

2. Digitale Strategie entwerfen und Ziele definieren: Nachdem Sie sich ein klares Bild verschafft haben, ist es wichtig, eine gezielte Digital-Leader-Strategie zu entwickeln. Hier ist die direkte Zusammenarbeit mit Ihren Mitarbeitern von Bedeutung. Mehr dazu findet sich im Artikel zur digitalen Strategie.

3. Klare Rahmenbedingungen erstellen: Jetzt geht es an die Arbeit - die Zusammenarbeit. Diese muss geregelt werden, aber nicht mit engen Richtlinien verknüpft werden. Eine moderne Führungskraft setzt Rahmenbedienungen für den Mitarbeiter. Mehr dazu im Artikel zum Agile Leadership.

4. Digitalkompetenz ausbauen: Nachdem eine Führungskraft sich bewusst gemacht hat, wie die digitale Transformation sich strategisch auf das Unternehmen auswirkt, sollte diese auch operativ mitwirken. Regelmäßige Workshops zu Themen der digitalen Transformation und die Einführung von Tools zur Unterstützung sind wichtig, um eine digitale Kultur zu schaffen. Lesen Sie dazu mehr zur Digital Culture.

5. Content und Image digital fördern: Wenn die Arbeit läuft und Sie in den Prozessen angekommen sind, dann gilt es die Zusammenarbeit als ein loyales, wachsames Netzwerk zu nutzen. Jetzt gilt es dieses 


\section{DIGITAL LEADERSHIP}

Wissen auf die Straße zu bringen und als Aushängeschild zu verwenden (Stichwort Content Marketing). Viele aufmerksame Köpfe können hier helfen. Lassen Sie Ihren Mitarbeitern aber Freiraum für eigene Ideen und Beiträge. Lesen Sie mehr im Artikel zum agilen Marketing.

6. Vorbilder etablieren: Erfolgreiche Führungskräfte im digitalen Zeitalter verändern Unternehmen, leben Offenheit vor und sind agil. Vorbilder, die diese Attribute in ihrer Führungsrolle beispielhaft umsetzen, werden als digitale Vorbilder wahrgenommen. Vorbilder können auch andere Mitarbeiter im Team sein, welche z. B. neue Tools direkt gewinnbringend nutzen. Lesen Sie mehr zum digitalen Leadership.

7. Pioniergeist etablieren: Gerade in einem Wandel passieren Fehler. Sie sind menschlich. „Fehler vermeidet man, indem man Erfahrung sammelt. Erfahrung sammelt man, indem man Fehler macht" (Laurence Johnston Peter). In unserer Gesellschaft ist es häufig so, dass Fehler negativ behaftet sind. Das fängt schon in der Schule an. Fehler sind "schlecht" und werden bestraft, Fehlerlosigkeit wird belohnt. Dies ist in der digitalen Transformation nicht mehr möglich. Durch hohe Komplexität sind wir gezwungen auszuprobieren und Fehler zuzulassen, um Neues zu erkunden. Lesen Sie mehr zum Thema Leading Digital.

\section{FAZIT}

Insgesamt haben sich die Anforderungen an die Führungskräfte in einer digitalen Welt verändert. Die unterschiedlichen Generationen (von Babyboomer bis Generation Z) müssen zu einem Team geformt und „digital ge- 


\section{DIGITAL LEADERSHIP}

führt“ werden. Es muss also ein Umdenken in den Köpfen von digitalen Managern stattfinden. Führungskräfte sind laut diesen Studien als Vorbilder und Begleiter gefordert.

Es ist jedoch klar, dass diese digitale Führung nicht unterschätzt werden sollte. Unternehmen, die begreifen, dass die eigene digitale Transformation von den Menschen im Unternehmen abhängig ist und genau darin investieren, werden es zukünftig einfacher haben. Führende Experten meinen also, dass Agilität und Innovation Teil der DNA eines jeden Unternehmens werden sollen. Mithilfe der sieben Tipps kann sicher ein Schritt in diese Richtung gegangen werden. Denn das Abenteuer Digitalisierung geht gerade erst los!

\section{QUELLEN}

https://www.de.capgemini-consulting.com/resource-file-

ac-

cess/resource/pdf/digital_leadership_fuhrungskrafteentwicklung_im_digita len_zeitalter_26.1_0.pdf

http://www.hannovermesse.de/de/news/digital-leadership-wie-der-digitalewandel-die-fuehrung-veraendert.xhtml

https://www.accenture.com/de-de/insight-digital-leaders-moving-into-fastlane 


\section{DIALOG MIT SVENJA HOFERT ZUM THEMA AGILERE UNTER- NEHMEN}

Originalartikel: https://agile-unternehmen.de/svenja-hofert-agilereunternehmen/

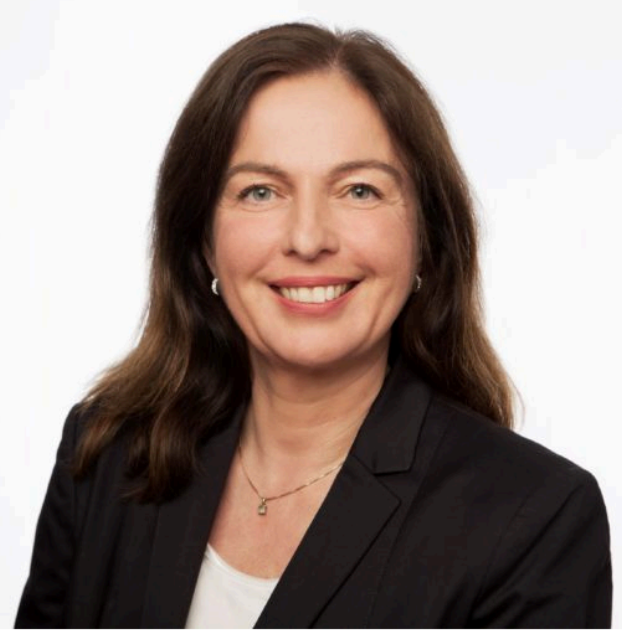

Nachdem ich im Zuge der Forschung zahlreiche Bücher und Quellen zitiert habe, war es mein Anliegen, auch die Gesichter der wichtigsten Publikationen kennenzulernen und mich mit diesem auszutauschen. So habe ich einen Dialog mit Svenja Hofert zum Thema Agilere Unternehmen geführt. Svenja Hofert ist Autorin des Buches Agiler führen: Einfache Maßnahmen für bessere Teamarbeit, mehr Leistung und höhere Kreativität. Svenja Hofert ist Geschäftsführerin der Karriereberatung Karriere \& Entwicklung und von Teamworks GTQ Gesellschaft für Teamentwicklung und Qualifizierung $\mathrm{mbH}$. Sie ist Unternehmerin, 


\section{DIGITAL LEADERSHIP}

Management Coach, Rednerin und Bloggerin. Im Dialog habe ich Frau Hofert einige spannende Thesen gegeben und fasse ihre Gedanken zu diesen im Artikel zusammen.

\section{NUR AGILE UNTERNEHMEN SIND ZUKUNFTSFÄHIG!}

Agilität ist seit paar Jahren ein totaler Hype, so Hofert. Das liegt einerseits an den Medien und andererseits an der digitalen Transformation. Unternehmen werden durch Startups und ständig ändernde Kundenwünsche bedroht. Sogar etablierte Konzerne sind schon lange nicht mehr unantastbar. Es gibt ebenfalls völlig neue Anforderungen an Innovationen. Kunden sind anspruchsvoller und Produkte müssen digital, individuell anpassbar und absolut revolutionär sein, damit Kunden sich noch an diesen erfreuen.

„Unsere Welt ist sehr unterschiedlich“, so Hofert. Viele Unternehmen mit sehr wenig Agilität sind aktuell ebenfalls sehr erfolgreich. Die Frage für Hofert lautet also: Wo steht das Unternehmen und wo will es hin? Auch eine Buchhaltung kann agil sein. Die Frage ist laut Hofert: Wie viel Sinn macht das? Aus diesem Grund vertritt Hofert den Ansatz, dass jedes Unternehmen bereits einen gewissen Grad an Agilität besitzt und man nur genau überlegen muss, mit welchen Methoden und wie weit man diese sinnvoll steigern kann. Das heißt konkret: „Agilere Unternehmen sind also zukunftsfähig.“

\section{NUR AGILERE UNTERNEHMEN SIND ZUKUNFTSFÄHIG!}

Der Titel von Hoferts Buch lautet: „Agiler führen“. Unter dem Aspekt haben wir über die Bedeutung dieser Meta- 


\section{DIGITAL LEADERSHIP}

pher gesprochen. Hofert definiert ihre Überlegungen nicht als fundamental neues Rahmenwerk oder einen neuen Management-Stil. Ihre Motivation ist es Unternehmen gezielt und sinnvoll zu verbessern und "reifer zu machen“.

Jedes Unternehmen verfügt bereits über einen gewissen Grad an Agilität und wir müssen nun entscheiden, wie diese noch gesteigert werden kann.

So existiert auf der Webseite von Frau Hofert ein Reifegradmodell für agile Unternehmen. Anhand der menschlichen Reife der Mitarbeiter und der Art der Führung definiert Frau Hofert also mehrere Stufen von agilen Unternehmen. So kann laut Hofert jedes Unternehmen den IstZustand feststellen und genau evaluieren, bis zu welchem Level dieser gesteigert werden sollte.

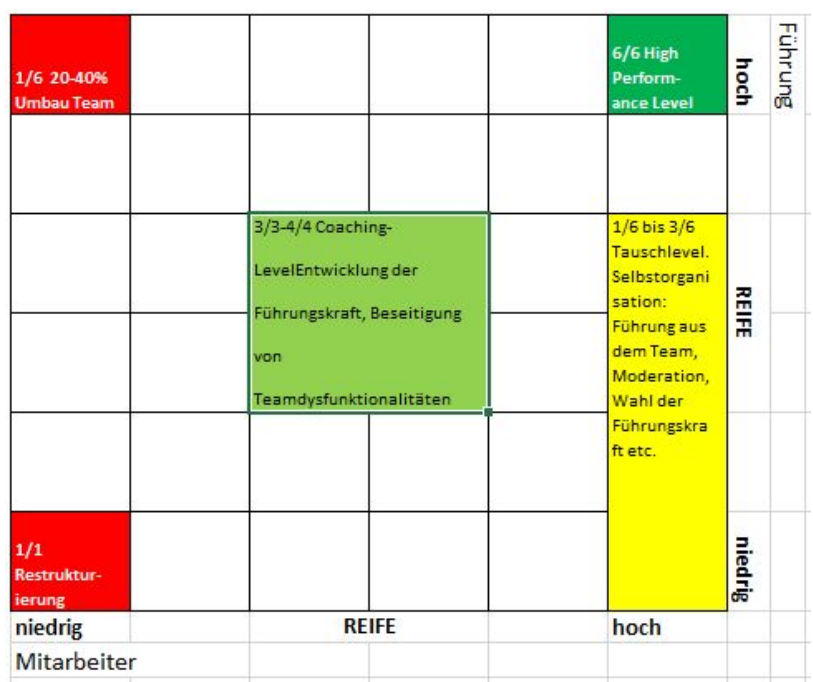




\section{DIGITAL LEADERSHIP}

Die agile Reifegradmatrix von Svenja Hofer zeigt agile Reife anhand von Führung und Mitarbeitern (Quelle: Svenja Hofert).

\section{WIR MÜSSEN FÜHRUNG NEU DENKEN!}

Ein großer Teil von Frau Hoferts Buch befasst sich mit Führung. Hier fordert sie auf, Führung „neu zu denken“. Hinter dieser These steht vor allem der Aspekt der Aufgabe von Command und Control und der Hinwendung zu Selbstorganisation, so Hofert.

Agiles Führen ist eine dynamische Haltung, ein Mindset, das Veränderung als Dauerzustand begreift. Agile Führungskräfte sind beweglich, flexibel und fähig zur Transformation von Menschen, Teams und Prozessen. Sie begreifen Führung als Rolle anstatt als Position oder Funktion.

Einen weiteren wichtigen Punkt fügte Frau Hofert direkt der Definition von agiler Führung hinzu: Agile Führungskräfte fördern die Selbstorganisation von Gruppen durch permanente Teamentwicklung. Ziel ist die Förderung von Selbstverantwortung und Kreativität. Dies sieht Hofert als einen essenziellen Skill von Führungskräften, da oft zu viel Wert auf die Entwicklung von einzelnen Personen statt Teams gesetzt wird. Die Entwicklung zu sogenannten High Performance Teams ist also eine wichtige Aufgabe einer agilen Führungskraft.

\section{HIGH PERFORMANCE TEAMS SIND ...}

... Teams, welche bereits eine überdurchschnittliche Leistung zu bringen, aber trotz dieser Leistung nicht damit 


\section{DIGITAL LEADERSHIP}

aufhören, diese weiter zu steigern, so Hofert. Sie führt dazu das Beispiel von Bayern München an, die trotz sehr guter Leistungen nicht damit aufhören, sich konstant weiter zu verbessern.

Ein weiterer Aspekt von solchen Teams ist laut Hofert, dass diese sich nicht lediglich mit dem eigenen Unternehmen vergleichen. Am Beispiel von Bayern München sagt Hofert: Der FC Bayern vergleicht sich nicht mit der Bundesliga, sondern mit anderen internationalen Top-Clubs. So zeigen außerdem verschiedene Studien, dass Teams, welche sich mit anderen externen Teams austauschen, oft eine weitaus höhere Leistung bringen.

\section{AGILERE TEAMS SIND ERFOLGREICHER ALS WENI- GER AGILE TEAMS!}

"Agile Teams sind sozialer und kommunizieren mehr", so Hofert. Das Ergebnis der Studie ist jedoch nicht überraschend. Was jedoch überrascht, ist, wie deutlich das Ergebnis ausfiel. In der Studie hat Hofert agile Teams an 6 Faktoren festgemacht:

1. Teamentscheidung, das heißt gemeinsame Entscheidungen der Kollegen, keine Weisung durch einen direkten disziplinarischen Vorgesetzten.

2. Tägliche strukturierte Meetings mit allen Teammitgliedern, zum Beispiel sogenannte Stand-upMeetings, Daily Scrum.

3. Iterative Planung, das heißt Planung in kurzen Zyklen, innerhalb derer das Team nicht durch neue 


\section{DIGITAL LEADERSHIP}

Anforderungen von außen (Kunden, Management) gestört werden darf.

4. Nutzung eines Boards zur Darstellung und Visualisierung des Workflows, das von jedem Teammitglied gesehen werden kann und den Arbeitsstand offen zeigt.

5. Eigenverantwortliche Zuteilung von Aufgabenpaketen.

6. Retrospektiven, also Teamtreffen mit dem Ziel, aus der Vergangenheit zu lernen und die Arbeit ex post zu analysieren.

Hofert hat die Teilnehmer der Umfrage mithilfe eines eigenen Fragebogens in die Kategorien agil und nicht-agil eingeordnet. Danach wurden diese mit dem Fragebogen "Teamklima-Inventar" nach West/Brodbeck befragt und die Unterschiede bei den Antworten untersucht. 5 Hypothesen konnten bestätigt werden - sowohl das Teamklima als auch die Subskalen Visionsorientierung, Aufgabenorientierung, partizipative Sicherheit und Innovationsbereitschaft waren bei den agil arbeitenden Teilnehmern signifikant größer als bei den nicht-agilen. Ich danke auch Frau Hofert recht herzlich für die Zeit, welche sie sich für mich genommen hat.

\section{QUELLEN}

http://teamworks-gmbh.de/agiles-reifegrad-grid-fuer-leadership-undorganisation/ 


\section{Nachwort}

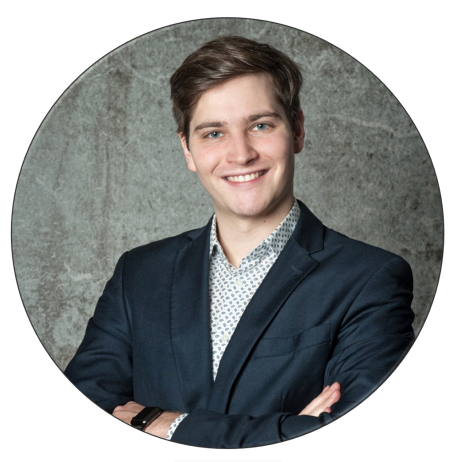

Zusammenfassend zeigt sich, dass der digitale Wandel bereits im vollen Gange ist und Unternehmen zunehmend verändert. Wir sind gerade erst dabei, uns dieser tiefgreifenden Änderungen bewusst zu werden und können die Folgen dieses Wandels aktuell nur erahnen.

Im Zuge meiner Forschung bin ich aktuell dabei, die Bereiche weitergehend $z u$ erforschen und werde alle Ergebnisse auf meinem Blog veröffentlichen.

Ich hoffe damit Ihr Interesse geweckt zu haben und Sie bald auf meiner Seite https://agile-unternehmen.de begrüßen zu dürfen!

\section{Dominic Lindner}




\section{Genderhinweis \& Bild- nachweis}

In diesem Blog werden so weit wie möglich geschlechtsneutrale Formulierungen verwendet. Wo dies nicht möglich ist, wird zur leichteren Lesbarkeit die männliche Form verwendet. Sofern keine explizite Unterscheidung getroffen wird, sind daher stets sowohl Frauen als auch Männer gemeint. Ebenso zeigen die Bilder auf der Webseite Frauen, Männer sowie Menschen unterschiedlicher Nationen und Herkunft. Jedes Bild wurde nach Relevanz ausgewählt und soll in keiner Weise Minderheiten diskriminieren. Es wurde jedoch versucht auf eine gerechte Verteilung zu achten.

- Successful businessman with bicycle @ Sergey Nivens

- Group of students in the café (c) luckybusiness

- Business people standing with a digital tablet (c) WavebreakmediaMicro

- Man of success in suit (c) Photographee.eu

- Business Team um Laptop Computer @ Robert Kneschke

- Manager with laptop and smart phone, London City Hall @ Halfpoint

- Group of young people @ georgerudy

- junge frau im Büro lehnt sich zufrieden zurück (c) contrastwerkstatt

- Break from work $\odot$ bernardbodo

- Young employee sitting outdoors @ olly

- Happy businessman sitting and using tablet on business meeting $\odot$ Drobot 


\section{Impressum}

Copyright: Mai 2017 - Dominic Lindner

Umschlaggestaltung, Illustration, Lektorat, Korrektorat: Dominic Lindner

Publisher: Dominic Lindner - Projektify e.V.

Das Werk, einschließlich seiner Teile, ist urheberrechtlich geschützt. Jedoch darf das Werk gern verbreitet werden. Auszüge sind nur unter Angabe einer Quelle zu verwenden. Dies gilt insbesondere für die elektronische oder sonstige Vervielfältigung, Übersetzung, Verbreitung und öffentliche Zugänglichmachung. Bei Fragen wenden Sie sich an:

Dominic.Lindner@agile-unternehmen.de 


\section{Zukunftsfähig in der digi- talen Transformation}

Wir leben in einer komplexen Welt mit schnellen und tiefgreifenden Veränderungen. Ein wesentlicher Treiber dieser ist die exponentielle technologische Entwicklung der letzten Jahre.

Ein Ansatz, um der Komplexität und Geschwindigkeit dieses Wandels entgegenzuwirken, könnte in einer gesteigerten Agilität bei bestimmten Schlüsselbereichen eines Unternehmens liegen.

In diesem E-Book möchte ich die Bereiche Führung, Organisation und Arbeit im Kontext dieses Megatrends genau beleuchten sowie den Einfluss auf Agilität darstellen. In jedem Kapitel findet sich eine Begriffserklärung, welche von der Klärung des Begriffs im Kontext des digitalen Wandels gefolgt ist sowie ein Dialog mit einem Experten wie Boris Gloger, Svenja Hofert, Niels Pfläging, Valentin Nowotny und Dr. Andreas Zeuch.

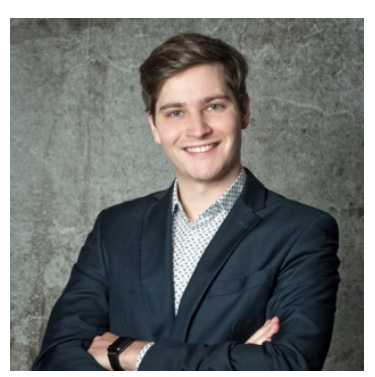

\section{Dominic Lindner}

Seit April 2016 führe ich den Blog agile-unternehmen.de und veröffentliche dort u. a. die Ergebnisse rund um meine Doktorarbeit. Diese absolviere ich nebenberuflich an der FAU Erlangen-Nürnberg. 